\title{
ON THE COMMUTABILITY OF HOMOGENIZATION AND LINEARIZATION IN FINITE ELASTICITY
}

\author{
STEFAN MÜLLER \& STEFAN NEUKAMM
}

\begin{abstract}
We consider a family of non-convex integral functionals $\frac{1}{h^{2}} \int_{\Omega} W(x / \varepsilon, \operatorname{Id}+h \nabla g(x)) \mathrm{d} x, \quad g \in W^{1, p}\left(\Omega ; \mathbb{R}^{n}\right)$

where $W$ is a Carathéodory function periodic in its first, and non-degenerate in its second variable. We prove under suitable conditions that the $\Gamma$-limits corresponding to linearization $(h \rightarrow 0)$ and homogenization $(\varepsilon \rightarrow 0)$ commute, provided $W$ is minimal at the identity and admits a quadratic Taylor expansion at the identity. Moreover, we show that the homogenized integrand, which is determined by a multi-cell homogenization formula, has a quadratic Taylor expansion with a quadratic term that is given by the homogenization of the second variation of $W$.
\end{abstract}

Keywords: homogenization, nonlinear elasticity, linearization, $\Gamma$-convergence.

2010 Mathematics Subject Classification: 35B27, 49J45, 74E30, 74Q05, 74Q20.

\section{INTRODUCTION}

In this contribution we consider periodic integral functionals of the type

$$
\int_{\Omega} W(x / \varepsilon, \nabla u(x)) \mathrm{d} x, \quad u \in W^{1, p}\left(\Omega ; \mathbb{R}^{n}\right)
$$

where $\varepsilon$ is a positive parameter, $\Omega$ an open, bounded Lipschitz domain in $\mathbb{R}^{n}$ with $n \geq 2$ and $W: \mathbb{R}^{n} \times \mathbb{M}^{n} \rightarrow[0,+\infty)$ is a Carathéodory function $Y:=[0,1)^{n}$-periodic in its first variable.

Functionals of this type model manifold situations in physics and engineering. We are particularly interested in applications to elasticity. In this context the integral in (1.1) is the elastic energy of a periodic composite material with period $\varepsilon$ deformed by the map $u: \Omega \rightarrow \mathbb{R}^{n}$. In situations where the deformation is close to a rigid deformation, say $|\nabla u-\mathrm{Id}| \sim h$, it is natural to introduce the scaled displacement $g(x):=h^{-1}(u(x)-x)$ and to consider the scaled, but equivalent energy

$$
\mathcal{I}^{\varepsilon, h}(g):=\frac{1}{h^{2}} \int_{\Omega} W(x / \varepsilon, \operatorname{Id}+h \nabla g(x)) \mathrm{d} x, \quad g \in W^{1, p}\left(\Omega ; \mathbb{R}^{n}\right) .
$$

For small parameters $\varepsilon$ and $h$ we expect that the energy $\mathcal{I}^{\varepsilon, h}$ can be replaced by an effective model that is simpler than the original one, but nevertheless captures the essential behavior from a macroscopic perspective. In this context the limit $h \rightarrow 0$ corresponds to linearization, while the theory of homogenization studies the

Date: October 9, 2018. 
asymptotic behavior of the energy as $\varepsilon \rightarrow 0$. Obviously, there are two orderings to derive an effective model by consecutively passing to the limits $\varepsilon \rightarrow 0$ and $h \rightarrow 0$. The goal of this paper is to show that both ways lead to the same limiting energy. In other words we prove that homogenization and linearization commute.

It is well-known that in the case where the integrand $W$ is convex in its second variable the homogenization of (1.1) is the integral functional $\int_{\Omega} W_{\mathrm{hom}}^{(1)}(\nabla u(x)) \mathrm{d} x$, where the homogenized integrand is given by the one-cell homogenization formula

$$
W_{\text {hom }}^{(1)}(F):=\inf \left\{\int_{Y} W(y, F+\nabla \varphi(y)) \mathrm{d} y: \varphi \in W_{\text {per }}^{1, p}\left(Y ; \mathbb{R}^{n}\right)\right\} .
$$

This result goes back to P. Marcellini [15] and was extensively studied with various methods (cf. e.g. [8], 2] ). The more general setting of monotone operators and nonperiodic dependency on the spatial variable was studied by L. Tartar [20, 11, 21. On the other side, for non-convex $W$ it turns out that in general the relaxation of $W$ over one - or even an ensemble of finitely many copies of the periodicity cell $Y$ is not sufficient for homogenization. Nevertheless, A. Braides [4, 5] and the first author [16] showed in the 1980s that in the non-convex case the homogenization of the functional in (1.1) leads to the multi-cell homogenization formula

$$
W_{\mathrm{hom}}^{(\mathrm{mc})}(F):=\inf _{k \in \mathbb{N}} \inf \left\{\frac{1}{k^{n}} \int_{k Y} W(y, F+\nabla \varphi(y)) \mathrm{d} y: \varphi \in W_{\mathrm{per}}^{1, p}\left(k Y ; \mathbb{R}^{n}\right)\right\} .
$$

We state this result in a precise manner in Theorem 6.1 below. The fact that the one-cell formula is not sufficient in nonlinear elasticity has been observed earlier in the engineering literature (see [1, 22]). These authors observed that instabilities arise even when the one-cell formula predicts no loss of stability. Later in [13] this has been systematically investigated.

In this contribution we are interested in integrands of the following type: For $a>0$ and $p \in(1, \infty)$ let $\mathcal{W}(a, p)$ denote the class of Carathéodory functions $W: \mathbb{R}^{n} \times \mathbb{M}^{n} \rightarrow[0, \infty)$ that are $Y$-periodic in the first variable and that satisfy the following conditions (W1) - (W4). We suppose that $W$ is locally Lipschitz continuous and satisfies standard growth conditions of order $p$ :

$$
\left\{\begin{array}{l}
\frac{1}{a}|F|^{p}-a \leq W(y, F) \leq a\left(1+|F|^{p}\right) \quad \text { and } \\
|W(y, F)-W(y, G)| \leq a\left(1+|F|^{p-1}+|G|^{p-1}\right)|F-G|
\end{array}\right.
$$

for all $F, G \in \mathbb{M}^{n}$ and a.e. $y \in \mathbb{R}^{n}$. We suppose that $F=\mathrm{Id}$ is a natural state and $W$ is non-degenerate, i.e.

$$
\begin{aligned}
& W(y, \mathrm{Id})=0 \quad \text { for a.e. } y \in \mathbb{R}^{n}, \\
& W(y, F) \geq \frac{1}{a} \operatorname{dist}^{2}(F, S O(n)) \quad \text { for all } F \in \mathbb{M}^{n} \text { and a.e. } y \in \mathbb{R}^{n} .
\end{aligned}
$$

Furthermore, we suppose that $W$ admits a quadratic Taylor expansion at Id in the sense that

$$
\exists Q \in \mathcal{Q}: \limsup _{\substack{G \rightarrow 0 \\ G \neq 0}} \operatorname{esssup}_{y \in \mathbb{R}^{n}} \frac{|W(y, \operatorname{Id}+G)-Q(y, G)|}{|G|^{2}}=0 .
$$

Here and below $\mathcal{Q}$ denotes the set of Carathéodory functions $Q: \mathbb{R}^{n} \times \mathbb{M}^{n} \rightarrow[0,+\infty)$ that are $Y$-periodic in the first variable, non-negative 
and quadratic in the second variable and bounded in the sense that

$$
\forall G \in \mathbb{M}^{n}: \operatorname{ess}_{y \in \mathbb{R}^{n}} Q(y, G) \leq c|G|^{2}
$$

for a suitable constant $c>0$.

Remarks. (1) In elasticity it is natural to assume that $W$ is frame indifferent, i.e.

$W(y, R F)=W(y, F) \quad$ for all $R \in S O(n), F \in \mathbb{M}^{n}$ and a.e. $y \in \mathbb{R}^{n}$.

We have not introduced this assumption explicitly, because it is not required in the proof. Nevertheless, the lower bound (W3) is motivated by frame indifference.

(2) (W2) \& (W3) are the main assumptions in our analysis. For energy densities representing composite materials, condition (W2) requires that each of the single components of the composite is stress free in a common reference configuration. Note that this rules out the application to prestressed composites, as considered in Section 7.2

(3) The combination of (W3) \& (W4) can be interpreted as a generalization of Hooke's law to the geometrically nonlinear setting, in the sense that both conditions together imply that for infinitesimal small strains the stress is proportional to the strain.

For $W \in \mathcal{W}(a, p)$ we study the behavior of the homogenized integrand $W_{\mathrm{hom}}^{(\mathrm{mc})}$ near the identity. Our first main result is the following:

Theorem 1.1. Let $W \in \mathcal{W}(a, p)$. Then

$$
\limsup _{\substack{G \rightarrow 0, G \neq 0}} \frac{\left|W_{\mathrm{hom}}^{(\mathrm{mc})}(\operatorname{Id}+G)-Q_{\mathrm{hom}}^{(1)}(G)\right|}{|G|^{2}}=0 .
$$

The statement remains valid if we drop condition (W1).

This means that whenever the integrand $W$ admits a quadratic expansion at Id with a quadratic term $Q$, then also the homogenized integrand $W_{\text {hom }}^{(\mathrm{mc})}$ has a quadratic expansion where the quadratic term is given by the homogenization of $Q$. Partial results in this direction under strong implicit assumptions on the minimizers for the cell problems have been obtained in [13].

Remark 1.2. Conditions (W3) \& (W4) imply that the quadratic integrand $Q(y, \cdot)$ is a positive semi-definite quadratic form for almost every $y \in \mathbb{R}^{n}$. More precisely, the non-degeneracy condition (W3) implies that $Q(y, \cdot)$ restricted to the subspace of symmetric $n \times n$ matrices is positive definite; in particular, we have

$$
Q(y, G) \geq \frac{1}{a}|\operatorname{sym} G|^{2} \quad \text { for all } G \in \mathbb{M}^{n} \text { and a.e. } y \in \mathbb{R}^{n} .
$$

In virtue of Korn's inequality this guarantees that the minimization problem

$$
\int_{Y} Q(y, G+\nabla \varphi(y)) \mathrm{d} y \quad \text { subject to } \int_{Y} \varphi \mathrm{d} y=0
$$

has a unique minimizer in $W_{\text {per }}^{1,2}\left(Y ; \mathbb{R}^{n}\right)$. If the energy density $W$ additionally satisfies (W2) and is frame indifferent, then the associated quadratic form $Q$ vanishes for skew symmetric matrices. In this case $Q$ and its homogenization $Q_{\text {hom }}$ are energy densities as they typically appear in linear elasticity. 
Theorem 1.1 shows that the expansion property (W4) is stable under homogenization for energy densities in $\mathcal{W}(a, p)$. The next result states that also the non-degeneracy condition is stable under homogenization:

Lemma 1.3. Let $W \in \mathcal{W}(a, p)$, then $W_{\mathrm{hom}}^{(\mathrm{mc})} \in \mathcal{W}\left(a^{\prime}, p\right)$ for a positive constant $a^{\prime}=a^{\prime}(a, n)$. Additionally, the map $W_{\mathrm{hom}}^{(\mathrm{mc})}: \mathbb{M}^{n} \rightarrow[0, \infty)$ is continuous and quasiconvex.

In the language of $\Gamma$-convergence Theorem 1.1 implies that the $\Gamma$-limits of $\left(\mathcal{I}^{\varepsilon, h}\right)$ corresponding to linearization and homogenization commute. In order to state this in a precise manner, let $\gamma$ be a closed subset of $\partial \Omega$ with positive $n$-1-dimensional Hausdorff-measure. We denote the space of scaled displacements that satisfy the Dirichlet boundary condition on $\gamma$ by

$$
\mathcal{A}_{\gamma}:=\left\{g \in W^{1,2}\left(\Omega ; \mathbb{R}^{n}\right): g=0 \text { on } \gamma\right\} .
$$

For simplicity we assume that $\gamma$ is regular enough to guarantee that $\mathcal{A}_{\gamma} \cap W^{1, \infty}\left(\Omega ; \mathbb{R}^{n}\right)$ is strongly dense in $\mathcal{A}_{\gamma}$. We consider the following functionals from $L^{2}\left(\Omega ; \mathbb{R}^{n}\right)$ to $[0,+\infty]$ :

$$
\begin{aligned}
& \mathcal{I}^{h, \varepsilon}(g):= \begin{cases}\frac{1}{h^{2}} \int_{\Omega} W(x / \varepsilon, \operatorname{Id}+h \nabla g(x)) \mathrm{d} x & \text { if } g \in \mathcal{A}_{\gamma} \\
+\infty & \text { else, }\end{cases} \\
& \mathcal{I}_{\text {hom }}^{h}(g): \begin{cases}\frac{1}{h^{2}} \int_{\Omega} W_{\mathrm{hom}}^{(\mathrm{mc})}(\mathrm{Id}+h \nabla g(x)) \mathrm{d} x & \text { if } g \in \mathcal{A}_{\gamma} \\
+\infty & \text { else, }\end{cases} \\
& \mathcal{I}_{\text {lin }}^{\varepsilon}(g):: \begin{cases}\int_{\Omega} Q(x / \varepsilon, \nabla g(x)) \mathrm{d} x & \text { if } g \in \mathcal{A}_{\gamma} \\
+\infty & \text { else, }\end{cases} \\
& \mathcal{I}^{0}(g):= \begin{cases}\int_{\Omega} Q_{\text {hom }}^{(1)}(\nabla g(x)) \mathrm{d} x & \text { if } g \in \mathcal{A}_{\gamma} \\
+\infty & \text { else. }\end{cases}
\end{aligned}
$$

Our second main result is the following:

Theorem 1.4. Let $W \in \mathcal{W}(a, p)$ with $p \geq 2$. Then the following diagram commutes

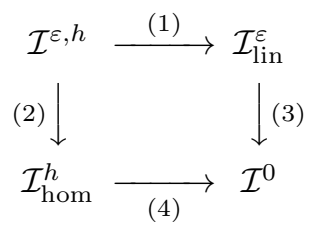

where (1),(4) and (2),(3) mean $\Gamma$-convergence with respect to strong convergence in $L^{2}\left(\Omega ; \mathbb{R}^{n}\right)$ as $h \rightarrow 0$ and $\varepsilon \rightarrow 0$, respectively.

In the diagram the $\Gamma$-limit (1), which corresponds to linearization of a heterogeneous energy, was treated by G. Dal Maso, M. Negri and D. Percivale in 9. In Section 5 we give a slight variant of their argument, which is adapted to assumption (W4). 
Remark 1.5. On the level of the energy functional $\mathcal{I}^{\varepsilon, h}$, it is natural to study also the $\Gamma$-convergence behavior as $\varepsilon$ and $h$ simultaneously converge to 0 . This corresponds to a "diagonal limit" in diagram (1.3). Indeed, in [17 the second author proves (based on two-scale convergence methods) that $\mathcal{I}^{\varepsilon, h} \Gamma$-converges to $\mathcal{I}^{0}$ as $(\varepsilon, h) \rightarrow(0,0)$ simultaneously. Theorem 1.4 is also related to recent works by A. Braides and B. Schmidt [, 19, where the passage from pair-interaction atomistic models to linear elasticity is studied. In contrast to the setting considered in the present paper, where $\varepsilon$ describes the length scale of the material heterogeneity, in [6, 19] the small scale $\varepsilon$ originates from the discrete nature of the atomistic model and measures the interatomic distance. In 19 the passage from-discreteto-continuous is obtained (in the regime $\varepsilon \ll h^{2}$ ) as a "diagonal limit", i.e. as a $\Gamma$-convergence result as $\varepsilon$ and $h$ simultaneously converge to 0 . It is observed that the derived $\Gamma$-limit coincides with the model obtained by linearizing the continuum model derived from the atomistic energy by applying the Cauchy-Born rule. The central assumption is a discrete version of the non-degeneracy condition (W3). In the atomistic setting this ensures that for sufficiently small displacements no oscillations on the length scale of the lattice emerge.

The $\Gamma$-convergence result is complemented by the following equi-coercivity statement:

Proposition 1.6. Suppose that $W \in \mathcal{W}(a, p)$ with $p \geq 2$. Then there exists a positive constant $c_{1}$ such that

$$
\min \left\{\mathcal{I}^{\varepsilon, h}(g), \mathcal{I}_{\text {lin }}^{\varepsilon}(g), \mathcal{I}_{\text {hom }}^{h}(g), \mathcal{I}^{0}(g)\right\} \geq c_{1} \Psi(g) .
$$

for all $\varepsilon, h>0$ and $g \in L^{2}\left(\Omega ; \mathbb{R}^{n}\right)$, where

$$
\Psi(g):= \begin{cases}\|g\|_{W^{1,2}\left(\Omega ; \mathbb{R}^{n}\right)}^{2} & \text { if } g \in \mathcal{A}_{\gamma} \\ +\infty & \text { else. }\end{cases}
$$

Remark 1.7. Obviously, the map $\Psi$ (and the restriction $\left.\Psi\right|_{\mathcal{A}_{\gamma}}$ ) is coercive and lower semicontinuous in $L^{2}\left(\Omega ; \mathbb{R}^{n}\right)$ (and coercive and lower semicontinuous with respect to weak convergence in $W^{1,2}\left(\Omega ; \mathbb{R}^{n}\right)$ respectively); thus, the previous proposition implies that the energies $\mathcal{I}^{\varepsilon, h}, \mathcal{I}_{\text {lin }}^{\varepsilon}, \mathcal{I}_{\text {hom }}^{h}$ and $\mathcal{I}^{0}$ are equi-coercive in the strong topology of $L^{2}\left(\Omega ; \mathbb{R}^{n}\right)$.

Remark 1.8. The local $p$-Lipschitz condition in (W1) can be dropped (see M. Baía and I. Fonseca [3]). Nevertheless, it is a natural property of quasiconvex or even rank-one convex functions: Let $p \in[1, \infty)$ and $f: \mathbb{M}^{n} \rightarrow[0, \infty)$ with $f(F) \leq$ $C\left(1+|F|^{p}\right)$. If $f$ is rank-one convex, then we have

$$
|f(F)-f(G)| \leq C\left(1+|F|^{p-1}+|G|^{p-1}\right)|F-G| .
$$

We would like to remark that the $p$-growth and coercivity condition is too restrictive for a direct application to finite elasticity, because we expect the behavior $W(y, F) \rightarrow+\infty$ as $F \rightarrow 0$ for realistic materials. In this direction, in [17 the second author considers periodic integrands $W$ that only satisfy (W2) - (W4). It turns out that Theorem 1.4 remains valid, when $\mathcal{I}_{\text {hom }}^{h}$ is replaced by the lower $\Gamma$-limit of $\left(\mathcal{I}^{\varepsilon, h}\right)_{\varepsilon}$ as $\varepsilon \rightarrow 0$.

The plan of the paper is as follows. In Section 2 we prove Lemma 1.3 and show that the non-degeneracy condition (W3) is stable under homogenization. In 
Section 3 we prove Proposition 1.6. In particular, we see that the non-degeneracy condition combined with the Dirichlet boundary condition imposed on $\gamma$ yield equicoercivity of the energies. The proof relies on an approach by G. Dal Maso et al. in [9] and combines the geometric rigidity estimate (see [12]) with an estimate on $\gamma$ that allows to eliminate free rotations (see 9 and Lemma 3.2 below). Moreover, we prove a simple rigidity estimate for periodic variations (see Lemma 3.4).

Section 4 is devoted to the proof of Theorem 1.1 and in Section 5 we present a linearization theorem that slightly extends results in [9] and is tailor-made for condition (W4). In Section 6 we discuss the diagram (1.3) and prove Theorem 1.4 Finally, in Section 7 we present two examples for which homogenization and linearization do not commute.

Notation. We denote the space of real $n \times n$-matrices by $\mathbb{M}^{n}$ and the subset of rotations, i.e. $F \in \mathbb{M}^{n}$ with $F^{\mathrm{T}} F=\mathrm{Id}$ and $\operatorname{det} F=1$, by $S O(n)$. For matrices $F, G \in \mathbb{M}^{n}$ we define the inner product and induced norm by

$$
\langle F, G\rangle:=\operatorname{tr} F^{\mathrm{T}} G \quad \text { and } \quad|F|:=\sqrt{\langle F, F\rangle} \quad \text { respectively. }
$$

Let $k \in \mathbb{N}$. A measurable function $u: \mathbb{R}^{n} \rightarrow \mathbb{R}$ is called $k Y$-periodic, if it satisfies $u(y+z)=u(y)$ for almost every $y \in \mathbb{R}^{n}$ and all $z \in k \mathbb{Z}^{n}$. We define the function spaces

$$
\begin{aligned}
L_{\mathrm{per}}^{p}(k Y) & :=\left\{u \in L_{\mathrm{loc}}^{p}\left(\mathbb{R}^{n}\right): u \text { is } k Y \text {-periodic }\right\}, \\
W_{\mathrm{per}}^{1, p}(k Y) & :=\left\{u \in L_{\mathrm{per}}^{p}(k Y): u \in W_{\mathrm{loc}}^{1, p}\left(\mathbb{R}^{n}\right)\right\}
\end{aligned}
$$

and likewise $L_{\text {per }}^{p}\left(k Y ; \mathbb{R}^{n}\right)$ and $W_{\text {per }}^{1, p}\left(k Y ; \mathbb{R}^{n}\right)$.

We denote the set of linear maps $\mathbb{L}$ from $\mathbb{M}^{n}$ to $\mathbb{M}^{n}$ that satisfy $\langle\mathbb{L} A, B\rangle=$ $\langle\mathbb{L} B, A\rangle$ for all $A, B \in \mathbb{M}^{n}$ by $\mathbb{T}_{\text {sym }}(n)$, i.e. $\mathbb{L} \in \mathbb{T}_{\text {sym }}(n)$ is a symmetric fourth order tensor. We associate to each quadratic integrand $Q \in \mathcal{Q}$ a unique map $\mathbb{L} \in L_{\text {per }}^{\infty}\left(Y ; \mathbb{T}_{\text {sym }}(n)\right)$ according to

$$
\langle\mathbb{L}(y) F, G\rangle=\frac{Q(y, F+G)-Q(y, F)-Q(y, G)}{2} \quad \text { for all } F, G \in \mathbb{M}^{n} .
$$

\section{Stability of the non-degeneracy condition. Proof of Lemma 1.3.}

In this section we prove Lemma 1.3. Let $W \in \mathcal{W}(a, p)$. It is well-known that the homogenized integrand $W_{\mathrm{hom}}^{(\mathrm{mc})}: \mathbb{M}^{n} \rightarrow[0, \infty)$ is a continuous and quasiconvex map that satisfies the $p$-growth and $p$-coercivity condition (W1) (see e.g. 16, 5] and [3]). Condition (W2) is trivially fulfilled and Theorem 1.1 implies that $W_{\mathrm{hom}}^{(\mathrm{mc})}$ has a Taylor expansion at Id, and therefore satisfies condition (W4). Thus, it remains to prove that the non-degeneracy condition (W3) is stable under homogenization. This is an immediate consequence of the following observation:

Lemma 2.1. Let $W: \mathbb{R}^{n} \times \mathbb{M}^{n} \rightarrow[0,+\infty]$ be a Carathéodory function $Y$-periodic in its first variable and suppose that $W$ satisfies the non-degeneracy condition (W3). Then for all $F \in \mathbb{M}^{n}$ we have

$$
W_{\text {hom }}^{(\mathrm{mc})}(F) \geq \frac{1}{a^{\prime}} \operatorname{dist}^{2}(F, S O(n))
$$

where the positive constant $a^{\prime}$ only depends on the dimension $n$ and the constant from condition (W3). 
Proof. By definition we can find for each $k \in \mathbb{N}$ a number $m_{k} \in \mathbb{N}$ and a map $\psi_{k} \in W_{\text {per }}^{1, p}\left(m_{k} Y ; \mathbb{R}^{n}\right)$ such that

$$
W_{\mathrm{hom}}^{(\mathrm{mc})}(F)+\frac{1}{k} \geq \frac{1}{m_{k}^{n}} \int_{m_{k} Y} W\left(y, F+\nabla \psi_{k}(y)\right) \mathrm{d} y .
$$

We apply the non-degeneracy condition (W3) to the right hand side and obtain

$$
W_{\mathrm{hom}}^{(\mathrm{mc})}(F)+\frac{1}{k} \geq \frac{1}{a} \frac{1}{m_{k}^{n}} \int_{m_{k} Y} \operatorname{dist}^{2}\left(F+\nabla \psi_{k}(y), S O(n)\right) \mathrm{d} y .
$$

By replacing the map $F \mapsto \operatorname{dist}^{2}(F, S O(n))$ by its quasiconvexification $\operatorname{Qdist}^{2}(\cdot, S O(n))$ we get a lower estimate:

$$
W_{\mathrm{hom}}^{(\mathrm{mc})}(F)+\frac{1}{k} \geq \frac{1}{a}\left(\frac{1}{m_{k}^{n}} \int_{m_{k} Y} \operatorname{Qdist}^{2}\left(F+\nabla \psi_{k}(y), S O(n)\right) \mathrm{d} y\right) .
$$

Because $m_{k} Y$ is a quadratic domain and $\psi_{k}$ is $m_{k} Y$-periodic, we see that the integral in the braces is bounded from below by $\operatorname{Qdist}^{2}(F, S O(n))$ due to quasiconvexity. Thus,

$$
W_{\mathrm{hom}}^{(\mathrm{mc})}(F)+\frac{1}{k} \geq \frac{1}{a} \operatorname{Qdist}^{2}(F, S O(n)) .
$$

K. Zhang proved in 23] that the quasiconvexification $\operatorname{Qdist}^{2}(\cdot, S O(n))$ can be bounded from below by $c_{n} \operatorname{dist}^{2}(\cdot, S O(n))$ where $c_{n}$ is a positive constant. Hence, we arrive at

$$
W_{\mathrm{hom}}^{(\mathrm{mc})}(F)+\frac{1}{k} \geq \frac{1}{a^{\prime}} \operatorname{dist}^{2}(F, S O(n))
$$

where $a^{\prime}:=\frac{c_{n}}{a}>0$. Passing to the limit $k \rightarrow \infty$ completes the proof.

\section{EQUi-COERCIVITY BASED ON GEOMETRIC RIGIDITY}

In this section we prove Proposition [1.6. First, we would like to remark that Proposition 1.6 is an equi-coercivity statement. For instance the proposition implies that whenever a family of scaled displacements $\left(g_{\varepsilon, h}\right)_{\varepsilon, h} \subset L^{2}\left(\Omega ; \mathbb{R}^{n}\right)$ has equibounded energy, i.e.

$$
\limsup _{(\varepsilon, h) \rightarrow(0,0)} \mathcal{I}^{\varepsilon, h}\left(g_{\varepsilon, h}\right)<\infty,
$$

then it is relatively compact in $L^{2}\left(\Omega ; \mathbb{R}^{n}\right)$ and we can extract a subsequence that strongly converges to a map $g \in \mathcal{A}_{\gamma}$ in $L^{2}\left(\Omega ; \mathbb{R}^{n}\right)$. If additionally each element of the sequence $\left(g_{\varepsilon, h}\right)$ has finite energy, then the construction of $\Psi$ reveals that the relative compactness also holds with respect to the weak topology in $W^{1,2}\left(\Omega ; \mathbb{R}^{n}\right)$. In some sense this observation a priori justifies the presentation of the scaled energy in terms of the scaled displacement.

A first step towards the proof of Proposition 1.6 is to show that the norm of a displacement gradient can be controlled by the associated energy. Because of the non-degeneracy condition (W3), we want to establish an estimate in the form

$$
\forall g \in \mathcal{A}_{\gamma}: \int_{\Omega}|\nabla g|^{2} \mathrm{~d} x \leq C \frac{1}{h^{2}} \int_{\Omega} \operatorname{dist}^{2}(\mathrm{Id}+h \nabla g(x), S O(n)) \mathrm{d} x
$$


for a constant $C$ that is independent of $h$. A key ingredient in the proof of this estimate is the geometric rigidity estimate by G. Friesecke, R.D. James and the first author:

Theorem 3.1 (Geometric rigidity estimate [12]). Let $U$ be a bounded Lipschitz domain in $\mathbb{R}^{n}, n \geq 2$. There exists a constant $C(U)$ with the following property: For each $v \in W^{1,2}\left(U ; \mathbb{R}^{n}\right)$ there is an associated rotation $R \in S O(n)$ such that

$$
\int_{U}|\nabla v(x)-R|^{2} \mathrm{~d} x \leq C(U) \int_{U} \operatorname{dist}^{2}(\nabla v(x), S O(n)) \mathrm{d} x .
$$

Moreover, the constant $C(U)$ is invariant under uniform scaling of $U$.

In virtue of this result we can assign to each $g \in \mathcal{A}_{\gamma}$ and positive parameter $h$ a single rotation $R \in S O(n)$ such that

$$
\left\|\frac{\operatorname{Id}+h \nabla g(x)-R}{h}\right\|_{L^{2}\left(\Omega ; \mathbb{M}^{n}\right)}^{2} \leq C(\Omega) \frac{1}{h^{2}} \int_{\Omega} \operatorname{dist}^{2}(\mathrm{Id}+h \nabla g(x), S O(n)) \mathrm{d} x .
$$

Let us assume for a moment that $R=\mathrm{Id}$. Then the previous estimate and the non-degeneracy of $W$ directly imply (3.1). In general a similar observation is valid: By taking the Dirichlet boundary condition imposed on $\gamma$ into account we can eliminate the free rotation. This has been shown by G. Dal Maso et al. in [9]. In particular, they proved the following result:

Lemma 3.2 (see Lemma 3.3 in [9]). Let $\Omega$ be an open and bounded Lipschitz domain in $\mathbb{R}^{n}$ and $\gamma$ a measurable subset of $\partial \Omega$ with positive $(n-1)$-dimensional Hausdorff measure. For $F \in \mathbb{M}^{n}$ define

$$
|F|_{\gamma}^{2}:=\min _{b \in \mathbb{R}^{n}} \int_{\gamma}|F x-b|^{2} \mathrm{~d} \mathcal{H}^{n-1}(x) .
$$

Then there exists a positive constant $C$ such that

$$
|F|^{2} \leq C|F|_{\gamma}^{2}
$$

for all matrices $F$ that belong to the union of the cone generated by $\operatorname{Id}-S O(n)$ and the set of skew symmetric matrices in $\mathbb{M}^{n}$.

In the lemma above $\mathcal{H}^{n-1}$ denotes the $(n-1)$-dimensional Hausdorff measure. With this result at hand we are in position to prove the proposition:

Proof of Proposition 1.6. It follows from the proof of Proposition 3.4 in 9] that

$$
\|g\|_{W^{1,2}\left(\Omega ; \mathbb{R}^{n}\right)}^{2} \leq c^{\prime} \frac{1}{h^{2}} \int_{\Omega} \operatorname{dist}^{2}(\operatorname{Id}+h \nabla g(x), S O(n)) \mathrm{d} x
$$

for all $g \in \mathcal{A}_{\gamma}$ and all $h \in(0,1)$. Here and below $c^{\prime}$ denotes a positive constant that may vary from line to line, but can be chosen only depending on $W$ and the geometry of $\Omega$ and $\gamma$. For the sake of completeness we briefly sketch the proof: In virtue of Theorem 3.1 we can assign to each $g \in \mathcal{A}_{\gamma}$ and $h \in(0,1)$ a rotation $R \in S O(n)$ such that

$$
\int_{\Omega}|\operatorname{Id}+h \nabla g(x)-R|^{2} \leq c^{\prime} \operatorname{dist}^{2}(\operatorname{Id}+h \nabla g(x), S O(n)) \mathrm{d} x .
$$


Since $h \nabla g=(\mathrm{Id}+h \nabla g-R)-(\mathrm{Id}-R)$, we have

$$
\|g\|_{W^{1,2}\left(\Omega ; \mathbb{R}^{n}\right)}^{2} \leq c^{\prime} \frac{1}{h^{2}} \int_{\Omega} \operatorname{dist}^{2}(\operatorname{Id}+h \nabla g(x), S O(n)) \mathrm{d} x+2\left\|\frac{R-\mathrm{Id}}{h}\right\|_{L^{2}\left(\Omega ; \mathbb{R}^{n}\right)}^{2} .
$$

Set $u(x):=(\operatorname{Id}-R) x+h g(x)-u_{\Omega}$ where $u_{\Omega} \in \mathbb{R}^{n}$ is chosen in such a way that $u$ is mean value free. Because $g(x)=0$ on $\gamma$, we have $u(x)=(\mathrm{Id}-R) x-u_{\Omega}$ on $\gamma$ and Lemma 3.2 implies that

$$
|\mathrm{Id}-R|^{2} \leq C|\mathrm{Id}-R|_{\gamma}^{2} \leq C \int_{\gamma}|u(x)|^{2} \mathrm{~d} \mathcal{H}^{n-1}(x) \leq c^{\prime} \int_{\Omega}|\nabla u(x)|^{2} \mathrm{~d} x,
$$

where we used the continuity of the trace operator and Poincaré-Wirtinger inequality. Because of the identity $\nabla u=\mathrm{Id}+h \nabla g-R$, the right hand side is controlled by $\int_{\Omega} \operatorname{dist}^{2}(\operatorname{Id}+h \nabla g(x), S O(n)) \mathrm{d} x$ and (3.2) follows.

By appealing to the non-degeneracy condition (W3) and Lemma 2.1, we immediately see that

$$
\min \left\{\mathcal{I}^{\varepsilon, h}(g), \mathcal{I}_{\text {hom }}^{h}(g)\right\} \geq c^{\prime} \frac{1}{h^{2}} \int_{\Omega} \operatorname{dist}^{2}(\operatorname{Id}+h \nabla g(x), S O(n)) \mathrm{d} x
$$

for all $g \in \mathcal{A}_{\gamma}$ and all $\varepsilon, h>0$. Since the energies are infinite whenever $g \notin \mathcal{A}_{\gamma}$, the previous estimate and (3.2) imply that

$$
\min \left\{\mathcal{I}^{\varepsilon, h}(g), \mathcal{I}_{\text {hom }}^{h}(g)\right\} \geq c^{\prime} \Psi(g) .
$$

for all $\varepsilon, h>0$ and $g \in L^{2}\left(\Omega ; \mathbb{R}^{n}\right)$.

Next, we consider the energies $\left(\mathcal{I}_{\text {lin }}^{\varepsilon}\right)$ and $\mathcal{I}^{0}$. It is not difficult to show that (W3) and (W4) imply that

$$
\min \left\{Q(y, F), Q_{\mathrm{hom}}^{(1)}(F)\right\} \geq c^{\prime}|\operatorname{sym} F|^{2} \quad \text { for all } F \in \mathbb{M}^{n} \text { and a.e. } y \in \mathbb{R}^{n} \text {. }
$$

Thus, it is sufficient to prove that

$$
\|g\|_{W^{1,2}\left(\Omega ; \mathbb{R}^{n}\right)}^{2} \leq c^{\prime} \int_{\Omega}|\operatorname{sym} \nabla g(x)|^{2} \mathrm{~d} x \quad \text { for all } g \in \mathcal{A}_{\gamma} .
$$

This can be seen as follows: Let $g \in \mathcal{A}_{\gamma}$. By Korn's inequality there exists a skew symmetric matrix $K \in \mathbb{M}^{n}$ such that

$$
\|\nabla g-K\|_{L^{2}\left(\Omega ; \mathbb{M}^{n}\right)}^{2} \leq c^{\prime} \int_{\Omega}|\operatorname{sym} \nabla g(x)|^{2} \mathrm{~d} x .
$$

We set $u(x):=g(x)-K x-u_{\Omega}$ where $u_{\Omega} \in \mathbb{R}^{n}$ is chosen in such a way that $u$ has vanishing mean value. As before we can apply Lemma 3.4 and find

$$
|K|^{2} \leq C|K|_{\gamma}^{2} \leq c^{\prime} \int_{\Omega}|\nabla u|^{2} \mathrm{~d} x .
$$

Since $\nabla u=\nabla g-K$, we find that

$$
\|g\|_{W^{1,2}\left(\Omega ; \mathbb{R}^{n}\right)} \leq c^{\prime}\|\nabla g\|_{L^{2}\left(\Omega ; \mathbb{M}^{n}\right)} \leq c^{\prime}\left(\|\nabla u\|_{L^{2}\left(\Omega ; \mathbb{M}^{n}\right)}+|K|\right) .
$$

Now (3.3) follows because the right hand side is controlled by $\|\operatorname{sym} \nabla g\|_{L^{2}\left(\Omega ; \mathbb{M}^{n}\right)}$. 
Remark 3.3. In the proof we did not use the property that $W$ satisfies the growth and coercivity condition (W1). Moreover, we could deduce from Theorem 1.4 and general properties of $\Gamma$-convergence, that $\left(\mathcal{I}_{\text {hom }}^{h}\right)$ and $\left(\mathcal{I}_{\text {lin }}^{\varepsilon}\right)$ and $\mathcal{I}^{0}$ are equi-coercive whenever $\left(\mathcal{I}^{\varepsilon, h}\right)$ is equi-coercive.

Another useful observation is the following:

Lemma 3.4. There exists a positive constant $c$ such that

$$
\frac{1}{h^{2}} \int_{k Y} \operatorname{dist}^{2}(\mathrm{Id}+h \nabla \psi(x), S O(n)) \mathrm{d} x \geq c \int_{k Y}|\nabla \psi|^{2} \mathrm{~d} y
$$

for all $h>0, k \in \mathbb{Z}$ and maps $\psi \in W_{\text {per }}^{1,2}\left(k Y ; \mathbb{R}^{n}\right)$.

Proof of Lemma 3.4. Set $v(x):=x+h \psi(x)$. By Theorem 3.1 there exists a rotation $R \in S O(n)$ satisfying

$$
\int_{k Y}\left|\frac{R-\mathrm{Id}}{h}-\nabla \psi(y)\right|^{2} \mathrm{~d} y \leq C \frac{1}{h^{2}} \int_{k Y} \operatorname{dist}^{2}(\mathrm{Id}+h \nabla \psi(y), S O(n)) \mathrm{d} y .
$$

The constant $C$ is independent of $h, k$ and $\psi$, because the domain $k Y$ is obtained by uniformly scaling the cell $Y$. We expand the left hand side and see that

$$
\int_{k Y}\left|\frac{R-\mathrm{Id}}{h}+\nabla \psi(y)\right|^{2} \mathrm{~d} y=\int_{k Y}\left|\frac{R-\mathrm{Id}}{h}\right|^{2}+2\left\langle\frac{R-\mathrm{Id}}{h}, \nabla \psi(y)\right\rangle+|\nabla \psi(y)|^{2} \mathrm{~d} y .
$$

Because gradients of functions in $W_{\text {per }}^{1,2}\left(k Y ; \mathbb{R}^{n}\right)$ are orthogonal to constant matrices (with respect to the standard inner product in $L^{2}\left(k Y ; \mathbb{M}^{n}\right)$ ), we deduce that the integral over the coupling term in the middle vanishes and we immediately obtain

$$
\int_{k Y}|\nabla \psi(y)|^{2} \mathrm{~d} y \leq C \frac{1}{h^{2}} \int_{k Y} \operatorname{dist}^{2}(\operatorname{Id}+h \nabla \psi(y), S O(n)) \mathrm{d} y .
$$

\section{Expansion of the multi-Cell homogenization formula. Proof of} THEOREM 1.1.

For convenience we define for $x \in \mathbb{R}^{n}$ and $G \in \mathbb{M}^{n} \backslash\{0\}$ the remainders

$$
\mathrm{r}(x, G):=\frac{W(x, \operatorname{Id}+G)-Q(x, G)}{|G|^{2}}
$$

and

$$
\mathrm{r}_{\mathrm{hom}}(G):=\frac{W_{\mathrm{hom}}^{(\mathrm{mc})}(\mathrm{Id}+G)-Q_{\mathrm{hom}}^{(1)}(G)}{|G|^{2}} .
$$

In order to prove Theorem 1.1] it is sufficient to show the following: For any sequence of matrices $\left(G_{k}\right)$ in $\mathbb{M}^{n} \backslash\{0\}$ with $\left|G_{k}\right| \rightarrow 0$ there holds

$$
\limsup _{k \rightarrow \infty} \mathrm{r}_{\text {hom }}\left(G_{k}\right)=0 \text {. }
$$

Because the normalized sequence $\left(\left|G_{k}\right|^{-1} G_{k}\right)$ is relatively compact, it is sufficient to consider sequences that additionally satisfy

$$
H_{k}:=\frac{G_{k}}{\left|G_{k}\right|} \rightarrow G \quad \text { in } \mathbb{M}^{n} \text { as } k \rightarrow \infty \text {. }
$$


In the sequel we separately prove that

$$
\begin{aligned}
& \limsup _{k \rightarrow 0} \frac{1}{\left|G_{k}\right|^{2}} W_{\mathrm{hom}}^{(\mathrm{mc})}\left(\mathrm{Id}+G_{k}\right) \leq Q_{\mathrm{hom}}^{(1)}(G), \\
& \liminf _{k \rightarrow 0} \frac{1}{\left|G_{k}\right|^{2}} W_{\mathrm{hom}}^{(\mathrm{mc})}\left(\mathrm{Id}+G_{k}\right) \geq Q_{\mathrm{hom}}^{(1)}(G) .
\end{aligned}
$$

Clearly, the validity of both estimates is equivalent to (4.2).

Step 1. We prove the upper bound estimate (4.4). Because $Q$ is a Carathéodory function quadratic in its second variable, the functional

$$
W_{\text {per }}^{1,2}\left(Y ; \mathbb{R}^{n}\right) \ni \varphi \mapsto \int_{Y} Q(y, G+\nabla \varphi(y)) \mathrm{d} y
$$

is lower semicontinuous with respect to weak convergence and continuous with respect to strong convergence. Furthermore, the strict convexity of $Q(x, \cdot)$ on the subspace of symmetric matrices is sufficient to guarantee that the functional admits a minimizer in $W_{\text {per }}^{1,2}\left(Y ; \mathbb{R}^{n}\right)$ (see Remark 1.2). The inclusion $C_{\text {per }}^{\infty}\left(Y ; \mathbb{R}^{n}\right) \subset$ $W_{\text {per }}^{1,2}\left(Y ; \mathbb{R}^{n}\right)$ is dense; thus, by the strong continuity of the functional we find for every $\eta>0$ a map $\psi \in C_{\text {per }}^{\infty}\left(Y ; \mathbb{R}^{n}\right)$ such that

$$
Q_{\mathrm{hom}}^{(1)}(G)=\min _{\varphi \in W_{\mathrm{per}}^{1,2}\left(Y ; \mathbb{R}^{n}\right)} \int_{Y} Q(y, G+\nabla \varphi(y)) \mathrm{d} y \geq \int_{Y} Q(y, G+\nabla \psi(y)) \mathrm{d} y-\eta .
$$

Based upon this choice we derive an upper bound for the left hand side in (5.5): By construction the multi-cell homogenization $W_{\mathrm{hom}}^{(\mathrm{mc})}(\cdot)$ is bounded from above by the one-cell homogenization $W_{\text {hom }}^{(1)}(\cdot)$; thus, we obtain

$$
W_{\mathrm{hom}}^{(\mathrm{mc})}\left(\mathrm{Id}+G_{k}\right) \leq W_{\mathrm{hom}}^{(1)}\left(\mathrm{Id}+G_{k}\right) \stackrel{(\star)}{\leq} \int_{Y} W\left(y, \mathrm{Id}+G_{k}+\left|G_{k}\right| \nabla \psi(y)\right) \mathrm{d} y .
$$

Inequality $(\star)$ follows from directly follows from the definition of $W_{\mathrm{hom}}^{(1)}$. We expand the integrand on the right hand side and deduce that for almost every $y \in Y$ we have

$$
\frac{1}{\left|G_{k}\right|^{2}} W\left(y, \mathrm{Id}+G_{k}+\left|G_{k}\right| \nabla \psi(y)\right) \leq Q\left(y, H_{k}+\nabla \psi(y)\right)+\mathrm{r}\left(y, G_{k}+\left|G_{k}\right| \nabla \psi(y)\right)
$$

where the remainder $\mathrm{r}$ is defined according to (4.1). Because $\left(G_{k}\right)$ vanishes and $\psi \in W^{1, \infty}\left(Y ; \mathbb{R}^{n}\right)$, we have

$$
G_{k}+\left|G_{k}\right| \nabla \psi(y) \rightarrow 0 \quad \text { uniformly; }
$$

thus, condition (W4) implies that

$$
\limsup _{k \rightarrow \infty} \int_{Y} \mathrm{r}\left(y, G_{k}+\left|G_{k}\right| \nabla \psi(y)\right) \mathrm{d} y=0 .
$$

Consequently, the previous estimates, the convergence $H_{k} \rightarrow G$ and the continuity of $Q$ lead to

$$
\limsup _{k \rightarrow \infty} \frac{1}{\left|G_{k}\right|^{2}} W_{\mathrm{hom}}^{(\mathrm{mc})}\left(\mathrm{Id}+G_{k}\right) \leq \int_{Y} Q(y, G+\nabla \psi(y)) \mathrm{d} y \leq Q_{\mathrm{hom}}^{(1)}(G)+\eta .
$$

Because $\eta>0$ can be chosen arbitrarily small, (5.5) follows. 
Step 2. We prove the lower bound estimate (4.5). We only have to consider the case where

$$
\liminf _{k \rightarrow \infty} \frac{1}{\left|G_{k}\right|^{2}} W_{\mathrm{hom}}^{(\mathrm{mc})}\left(\mathrm{Id}+G_{k}\right)
$$

is finite. We pass to a subsequence, that we do not relabel, such that

$$
\liminf _{k \rightarrow 0} \frac{1}{\left|G_{k}\right|^{2}} W_{\text {hom }}^{(\mathrm{mc})}\left(\mathrm{Id}+G_{k}\right)=\limsup _{k \rightarrow 0} \frac{1}{\left|G_{k}\right|^{2}} W_{\mathrm{hom}}^{(\mathrm{mc})}\left(\mathrm{Id}+G_{k}\right) .
$$

By definition, for all $k \in \mathbb{N}$ there exist a number $m_{k} \in \mathbb{N}$ and a map

$$
\psi_{k} \in W_{\mathrm{per}}^{1,2}\left(m_{k} Y ; \mathbb{R}^{n}\right) \quad \text { with } \int_{m_{k} Y} \psi_{k}(y) \mathrm{d} y=0
$$

such that

$$
W_{\text {hom }}^{(\mathrm{mc})}\left(\mathrm{Id}+G_{k}\right)+\frac{\left|G_{k}\right|^{2}}{k} \geq \frac{1}{m_{k}^{n}} \int_{m_{k} Y} W\left(y, \operatorname{Id}+G_{k}+\left|G_{k}\right| \nabla \psi_{k}(y)\right) \mathrm{d} y .
$$

This suggests to establish the lower bound estimate by applying the expansion in condition (W4) to the integral on the right hand side. Clearly, if $\left|\nabla \psi_{k}(y)\right|$ was bounded by a constant independent of $y$ and $k$, condition (W4) would immediately imply (4.5). However, the sequence $\left(\nabla \psi_{k}\right)$ is only bounded in the following sense:

$$
C:=\limsup _{k \rightarrow \infty} \frac{1}{m_{k}^{n}} \int_{m_{k} Y}\left|\nabla \psi_{k}\right|^{2} \mathrm{~d} y<\infty,
$$

as can be seen by appealing to the non-degeneracy condition (W3) and Lemma 3.4 Therefore, we distinguish points $y \in m_{k} Y$ where $\left|\nabla \psi_{k}(y)\right|$ is sufficiently small from those where $\left|\nabla \psi_{k}(y)\right|$ is too large for an expansion. More precisely, we define the set

$$
Y_{k}:=\left\{y \in m_{k} Y:\left|\nabla \psi_{k}(y)\right| \leq\left|G_{k}\right|^{-1 / 2}\right\}
$$

and denote the associated indicator function by $\chi_{k}$. The proof of the lower bound estimate is divided in two steps. First, we show that

$$
\liminf _{k \rightarrow \infty} \frac{1}{\left|G_{k}\right|^{2}} W_{\mathrm{hom}}^{(\mathrm{mc})}\left(\mathrm{Id}+G_{k}\right) \geq \liminf _{k \rightarrow \infty} \frac{1}{m_{k}^{n}} \int_{m_{k} Y} Q\left(y, \chi_{k}\left(H_{k}+\nabla \psi_{k}\right)\right) \mathrm{d} y
$$

and in a second step, we prove that

$$
\liminf _{k \rightarrow \infty} \frac{1}{m_{k}^{n}} \int_{m_{k} Y} Q\left(y, \chi_{k}\left(H_{k}+\nabla \psi_{k}\right)\right) \mathrm{d} y-Q_{\mathrm{hom}}^{(1)}(G) \geq 0 .
$$

It is obvious that the combination of both estimates justifies (4.5).

Step 3. (Proof of (4.9)). By construction we have

$$
\underset{y \in Y_{k}}{\operatorname{ess} \sup _{0}}\left|G_{k}+\right| G_{k}\left|\nabla \psi_{k}(y)\right| \rightarrow 0
$$

and in view of condition (W4) we see that

$$
\limsup _{k \rightarrow 0} \frac{1}{m_{k}^{n}} \int_{m_{k} Y} \chi_{k}\left|\frac{W\left(y, \mathrm{Id}+G_{k}+\left|G_{k}\right| \nabla \psi_{k}\right)}{\left|G_{k}\right|^{2}}-Q\left(y, H_{k}+\nabla \psi_{k}\right)\right| \mathrm{d} y=0 .
$$


Now the non-negativity of $W$ implies that $W(y, F) \geq \chi_{k}(y) W(y, F)$ for almost every $y \in m_{k} Y$ and all $F \in \mathbb{M}^{n}$; thus, estimate (4.6) and (4.11) immediately imply that

$$
\liminf _{k \rightarrow \infty} \frac{1}{\left|G_{k}\right|^{2}} W_{\text {hom }}^{(\mathrm{mc})}\left(\mathrm{Id}+G_{k}\right) \geq \liminf _{k \rightarrow \infty} \frac{1}{m_{k}^{n}} \int_{m_{k} Y} \chi_{k} Q\left(y, H_{k}+\nabla \psi_{k}\right) \mathrm{d} y .
$$

Because $\chi_{k}$ takes only values in $\{0,1\}$, we see that

$$
\chi_{k}(y) Q\left(y, H_{k}+\nabla \psi_{k}(y)\right)=Q\left(y, \chi_{k}(y)\left(H_{k}+\nabla \psi_{k}(y)\right)\right)
$$

for all $y \in m_{k} Y$ and (4.9) follows.

Step 4. (Proof of (4.10)). This is the heart of the matter. Obviously, if the indicator function $\chi_{k}$ was equal to 1 , then the integral in (4.10) would be bounded from below by $Q_{\mathrm{hom}}^{(1)}\left(H_{k}\right)$ and the estimate would follow from the continuity of $Q_{\mathrm{hom}}^{(1)}$. The general case would follow if we knew that

$$
\frac{1}{m_{k}^{n}} \int_{m_{k} Y}\left(1-\chi_{k}\right)\left|H_{k}+\nabla \psi_{k}\right|^{2} \mathrm{~d} x \rightarrow 0 .
$$

Because a priori $\left|\nabla \psi_{k}\right|^{2}$ could concentrate on the set where $\chi_{k}=0$, this is not obvious at all. Since we aim for a lower bound, we expand $Q\left(y, H_{k}+\nabla \psi_{k}\right)$ around the minimizer $G+\nabla \psi_{G}$ (see below). Then the most dangerous quadratic term has a sign.

Thus, let $\psi_{G} \in W_{\text {per }}^{1,2}\left(Y ; \mathbb{R}^{n}\right)$ satisfy

$$
Q_{\mathrm{hom}}^{(1)}(G)=\int_{Y} Q\left(y, G+\nabla \psi_{G}(y)\right) \mathrm{d} y .
$$

We extend $\psi_{G}$ by periodicity to $\mathbb{R}^{n}$. Since $Q$ is $Y$-periodic and convex, it is not difficult to check that $\psi_{G}$ is also a minimizer of the functional

$$
W_{\text {per }}^{1,2}\left(m_{k} Y ; \mathbb{R}^{n}\right) \ni \psi \mapsto \int_{m_{k} Y} Q(y, G+\nabla \psi(y)) \mathrm{d} y .
$$

Because $Q(y, \cdot)$ is a quadratic form, the inequality

$$
Q(y, A)-Q(y, B) \geq 2\langle\mathbb{L}(y)(A-B), B\rangle
$$

is valid for all $A, B \in \mathbb{M}^{n}$ and almost every $y$. We apply this inequality with

$$
A=\chi_{k}(y)\left(H_{k}+\nabla \psi_{k}(y)\right) \quad \text { and } \quad B=G+\nabla \psi_{G}(y) .
$$

Now integration over $m_{k} Y$ leads to

$$
\begin{aligned}
\frac{1}{2 m_{k}^{n}} \int_{m_{k} Y} Q\left(y, \chi_{k}\left(H_{k}+\nabla \psi_{k}\right)\right)-Q\left(y, G+\nabla \psi_{G}\right) \mathrm{d} y \\
\quad \geq \frac{1}{m_{k}^{n}} \int_{m_{k} Y}\left\langle\mathbb{L}(y)\left[\left(\chi_{k}\left(H_{k}+\nabla \psi_{k}\right)-\left(G+\nabla \psi_{G}\right)\right], G+\nabla \psi_{G}\right\rangle \mathrm{d} y .\right.
\end{aligned}
$$


The right hand side can be rewritten as

$$
\begin{aligned}
& \frac{1}{m_{k}^{n}} \int_{m_{k} Y}\left\langle\mathbb{L}(y)\left(H_{k}-G\right), G+\nabla \psi_{G}\right\rangle \mathrm{d} y \\
+ & \frac{1}{m_{k}^{n}} \int_{m_{k} Y}\left\langle\mathbb{L}(y)\left(\nabla \psi_{k}-\nabla \psi_{G}\right), G+\nabla \psi_{G}\right\rangle \mathrm{d} y \\
& -\frac{1}{m_{k}^{n}} \int_{m_{k} Y}\left\langle\mathbb{L}(y)\left(1-\chi_{k}\right)\left(H_{k}+\nabla \psi_{k}\right), G+\nabla \psi_{G}\right\rangle \mathrm{d} y \\
= & I_{k}^{(1)}+I_{k}^{(2)}+I_{k}^{(3)} .
\end{aligned}
$$

In the following we prove that all three integrals vanish as $k \rightarrow \infty$. We start with the first integral $I_{k}^{(1)}$. Due to the $Y$-periodicity of $\mathbb{L}$ and $\psi_{G}$, we have

$$
I_{k}^{(1)}=\int_{Y}\left\langle\mathbb{L}(y)\left(H_{k}-G\right), G+\nabla \psi_{G}\right\rangle \mathrm{d} y
$$

and (4.3) implies that $I_{k}^{(1)} \rightarrow 0$ as $k \rightarrow \infty$. We consider the second integral $I_{k}^{(2)}$. It is easy to check that $I_{k}^{(2)}$ is exactly the Euler Lagrange equation of the quadratic minimization problem associated to the functional (4.12). Because $\psi_{G}$ is a minimizer and the map $y \mapsto \psi_{k}-\psi_{G}$ an admissible test function, we have $I_{k}^{(2)}=0$ for all $k \in \mathbb{N}$.

Finally, we consider the third integral $I_{k}^{(3)}$. By applying the Cauchy-Schwarzand Hölder-inequality, we find that

$$
\left|I_{k}^{(3)}\right|^{2} \leq c^{\prime}\left(\frac{1}{m_{k}^{n}} \int_{m_{k} Y}\left|H_{k}+\nabla \psi_{k}\right|^{2} \mathrm{~d} y\right)\left(\frac{1}{m_{k}^{n}} \int_{m_{k} Y}\left|\left(1-\chi_{k}\right)\left(G+\nabla \psi_{G}\right)\right|^{2} \mathrm{~d} y\right)
$$

where $c^{\prime}$ is a positive constant independent of $k$. In virtue of (4.7), it is sufficient to prove that the second integral vanishes as $k \rightarrow \infty$. By construction the multi-cell $m_{k} Y$ is the disjoint union of the $m_{k}^{n}$ translated cells $Y+\xi$ with $\xi \in Z_{k}:=m_{k} Y \cap \mathbb{Z}^{n}$. Consequently, we can rewrite the second integral in (4.13) according to

$$
\begin{aligned}
\frac{1}{m_{k}^{n}} \int_{m_{k} Y}\left|\left(1-\chi_{k}\right)\left(G+\nabla \psi_{G}\right)\right|^{2} \mathrm{~d} y & \\
& =\frac{1}{m_{k}^{n}} \sum_{\xi \in Z_{k}} \int_{Y}\left(1-\chi_{k}(y+\xi)\right)\left|G+\nabla \psi_{G}(y+\xi)\right|^{2} \mathrm{~d} y
\end{aligned}
$$

where we used the fact that the map $y \mapsto\left(1-\chi_{k}\right)$ only takes values in $\{0,1\}$. Because the map $\psi_{G}$ is $Y$-periodic, the right hand side simplifies to

$$
\int_{Y} \bar{\chi}_{k}(y)\left|G+\nabla \psi_{G}(y)\right|^{2} \mathrm{~d} y \quad \text { with } \quad \bar{\chi}_{k}(y):=\frac{1}{m_{k}^{n}} \sum_{\xi \in Z_{k}}\left(1-\chi_{k}(y+\xi)\right) .
$$


Recall that $\chi_{k}$ denotes the indicator function of the set $Y_{k}$ defined in (4.8). By definition there holds $1 \leq\left|G_{k}\right|\left|\nabla \psi_{k}(y)\right|^{2}$ for a.e. $y \in m_{k} Y \backslash Y_{k}$. Thus, we estimate

$$
\int_{Y}\left|\bar{\chi}_{k}(y)\right| \mathrm{d} y \leq \frac{1}{m_{k}^{n}} \int_{m_{k} Y}\left(1-\chi_{k}(y)\right) \mathrm{d} y \leq \frac{1}{m_{k}^{n}} \int_{m_{k} Y}\left|G_{k}\right|\left|\nabla \psi_{k}(y)\right|^{2} \mathrm{~d} y \leq C\left|G_{k}\right|
$$

where $C$ denotes the constant from (4.7). Consequently, the sequence $\bar{\chi}_{k}$ strongly converges to 0 in $L^{1}(Y)$. We claim that

$$
\bar{\chi}_{k}\left|G+\nabla \psi_{G}\right|^{2} \rightarrow 0 \quad \text { strongly in } L^{1}(Y) .
$$

Since $\bar{\chi}_{k} \rightarrow 0$ in $L^{1}(Y)$, the left hand side converges to 0 in measure. On the other hand, by construction we have $0 \leq \bar{\chi}_{k} \leq 1$, and therefore the left hand side is dominated by the map $\left|G+\nabla \psi_{G}(\cdot)\right|^{2}$ which belongs to $L^{1}(Y)$. Thus, by dominated convergence assertion (4.14) follows and $I_{k}^{(3)}$ vanishes as $k \rightarrow 0$. So far we have shown that

$$
\frac{1}{m_{k}^{n}} \int_{m_{k} Y} Q\left(y, \chi_{k}\left(H_{k}+\nabla \psi_{k}\right)\right)-Q\left(y, G+\nabla \psi_{G}(y)\right) \mathrm{d} y \rightarrow 0
$$

as $k \rightarrow \infty$. In virtue of the $Y$-periodicity and convexity of $Q$, we have

$$
Q_{\mathrm{hom}}^{(1)}(G)=\frac{1}{m_{k}^{n}} \int_{m_{k} Y} Q\left(y, G+\nabla \psi_{G}(y)\right) \mathrm{d} y
$$

and (4.10) follows.

\section{LiNEARIZATION.}

G. Dal Maso et al. proved in 9] that linearized elasticity can be obtained as a $\Gamma$-limit from nonlinear, three-dimensional elasticity. The following theorem is a variant of their result adapted to assumption (W4).

Theorem 5.1. Let $W: \Omega \times \mathbb{M}^{n} \rightarrow[0, \infty)$ be a Carathéodory function and suppose that

$$
\limsup _{\substack{G \rightarrow 0, G \neq 0}} \frac{|W(x, \operatorname{Id}+G)-Q(x, G)|}{|G|^{2}}=0,
$$

where $Q: \Omega \times \mathbb{M}^{n} \rightarrow[0, \infty)$ is a Carathéodory function quadratic in its second variable and bounded in the sense that

$$
\forall G \in \mathbb{M}^{n}: \operatorname{ess}_{x \in \Omega} Q(x, G) \leq c_{1}|G|^{2}
$$

for a suitable constant $c_{1}>0$. We consider the functional

$$
\mathcal{E}^{h}(g):= \begin{cases}\frac{1}{h^{2}} \int_{\Omega} W(x, \operatorname{Id}+h \nabla g(x)) \mathrm{d} x & \text { if } g \in \mathcal{A}_{\gamma} \\ +\infty & \text { else, }\end{cases}
$$

and assume that there exists a positive constant $c_{2}$ such that

$$
\mathcal{E}^{h}(g) \geq c_{2}\|g\|_{W^{1,2}\left(\Omega ; \mathbb{R}^{n}\right)}^{2} \quad \text { for all } h>0 \text { and } g \in \mathcal{A}_{\gamma} .
$$


Then the family $\left(\mathcal{E}_{h}\right) \Gamma$-converges with respect to strong convergence in $L^{2}\left(\Omega ; \mathbb{R}^{n}\right)$ to the functional

$$
\mathcal{E}_{\operatorname{lin}}(g):= \begin{cases}\frac{1}{h^{2}} \int_{\Omega} Q(x, \nabla g(x)) & \text { if } g \in \mathcal{A}_{\gamma} \\ +\infty & \text { else. }\end{cases}
$$

Remark 5.2. Condition (5.2) implies equi-coercivity of the functionals $\left(\mathcal{E}^{h}\right)$ in $L^{2}\left(\Omega ; \mathbb{R}^{n}\right)$. In virtue of Proposition 1.6. we see that the combination of the nondegeneracy of $W$ and the Dirichlet boundary condition is a sufficient condition for (5.2).

Proof. Since the strong topology of $L^{2}\left(\Omega ; \mathbb{R}^{n}\right)$ is metrizable, we can use the sequential characterization of $\Gamma$-convergence. Thus, we have to prove the following:

(1) (lower bound) for every $g \in L^{2}\left(\Omega ; \mathbb{R}^{n}\right)$ and every sequence $\left(g_{h}\right)$ converging to $g$ in $L^{2}\left(\Omega ; \mathbb{R}^{n}\right)$ it is

$$
\liminf _{h \rightarrow 0} \mathcal{E}^{h}\left(g_{h}\right) \geq \mathcal{E}_{\operatorname{lin}}(g) .
$$

(2) (recovery sequence) for every $g \in L^{2}\left(\Omega ; \mathbb{R}^{n}\right)$ there exists a sequence $\left(g_{h}\right)$ converging to $g$ in $L^{2}\left(\Omega ; \mathbb{R}^{n}\right)$ such that

$$
\lim _{h \rightarrow 0} \mathcal{E}^{h}\left(g_{h}\right)=\mathcal{E}_{\operatorname{lin}}(g) .
$$

Step 1. (Recovery sequence). We only have to consider the case $g \in \mathcal{A}_{\gamma}$. By assumption the inclusion $\left(W^{1, \infty}\left(\Omega ; \mathbb{R}^{n}\right) \cap \mathcal{A}_{\gamma}\right) \subset \mathcal{A}_{\gamma}$ is dense; thus, there exists a sequence $\left(g_{h}\right)$ in $W^{1, \infty}\left(\Omega ; \mathbb{R}^{n}\right) \cap \mathcal{A}_{\gamma}$ that strongly converges to $g$ and that satisfies

$$
\underset{x \in \Omega}{\operatorname{essup}}\left|\nabla g_{h}(x)\right| \leq \frac{1}{\sqrt{h}} \quad \text { for all } h>0 .
$$

Thus, by (5.1) we get

$$
\frac{1}{h^{2}} \int_{\Omega} W\left(x, \operatorname{Id}+h \nabla g_{h}(x)\right) \mathrm{d} x=\int_{\Omega} Q\left(y, \nabla g_{h}(x)\right)+\mathrm{r}\left(x, h \nabla g_{h}(x)\right) \mathrm{d} x
$$

with

$$
\lim _{h \rightarrow 0}\left|\int_{\Omega} \mathrm{r}\left(x, h \nabla g_{h}(x)\right) \mathrm{d} x\right|=0 .
$$

Since the quadratic integral functional in (5.4) is continuous with respect to strong convergence and $g_{h} \rightarrow g$ strongly in $W^{1,2}\left(\Omega ; \mathbb{R}^{n}\right)$, we see that

$$
\lim _{h \rightarrow 0} \mathcal{E}^{h}\left(g_{h}\right)=\mathcal{E}_{\operatorname{lin}}(g) .
$$

Step 2. (Lower bound). Let $g \in L^{2}\left(\Omega ; \mathbb{R}^{n}\right)$ and $\left(g_{h}\right)$ a sequence converging to $g$

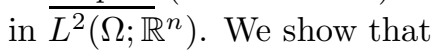

$$
\liminf _{h \rightarrow 0} \mathcal{E}^{h}\left(g_{h}\right) \geq \mathcal{E}_{\operatorname{lin}}(g) .
$$

As usual it is sufficient to consider the case where

$$
\liminf _{h \rightarrow 0} \mathcal{E}^{h}\left(g_{h}\right)=\limsup _{h \rightarrow 0} \mathcal{E}^{h}\left(g_{h}\right)<+\infty .
$$

In this case we can assume without loss of generality that each $g_{h}$ belongs to the set $\mathcal{A}_{\gamma}$. The equi-coercivity condition on $\left(\mathcal{E}^{h}\right)$ (see (5.2)) implies that $\left(g_{h}\right)$ is relatively 
compact with respect to weak convergence in $W^{1,2}\left(\Omega ; \mathbb{R}^{n}\right)$. As a consequence we can pass to a subsequence (that we do not relabel) such that

$$
g_{h} \rightarrow g \quad \text { weakly in } W^{1,2}\left(\Omega ; \mathbb{R}^{n}\right) .
$$

Because $\mathcal{A}_{\gamma}$ is a (seq.) weakly closed subset of $W^{1,2}\left(\Omega ; \mathbb{R}^{n}\right)$, we find that $g \in \mathcal{A}_{\gamma}$.

We define the set

$$
\Omega_{h}:=\left\{x \in \Omega:\left|\nabla g_{h}(x)\right| \leq h^{-1 / 2}\right\}
$$

and denote the corresponding indicator function by $\chi_{h}$. Since $\left(\nabla g_{h}\right)$ is a bounded sequence in $L^{2}\left(\Omega ; \mathbb{M}^{n}\right)$, we can estimate the measure of $\Omega \backslash \Omega_{h}$ according to

$$
\left|\Omega \backslash \Omega_{h}\right| \leq h \int_{\Omega \backslash \Omega_{h}}\left|\nabla g_{h}(x)\right|^{2} \mathrm{~d} x \leq h C
$$

for a suitable constant $C$. Now we establish a lower estimate by utilizing the expansion of $W$ for points $x \in \Omega_{h}$ and the non-negativity of $W$ for points $x \in \Omega \backslash \Omega_{h}$ :

$$
\int_{\Omega} W\left(x, \operatorname{Id}+h \nabla g_{h}(x)\right) \mathrm{d} x \geq \int_{\Omega_{h}} Q\left(x, \nabla g_{h}(x)\right)+\mathrm{r}\left(x, h \nabla g_{h}(x)\right) \mathrm{d} x .
$$

Since $\left|h \nabla g_{h}(x)\right| \leq \sqrt{h}$ for all $x \in \Omega_{h}$ the properties of the quadratic expansion of $W$ lead to

$$
\liminf _{h \rightarrow 0} \int_{\Omega} W\left(x, \operatorname{Id}+h \nabla g_{h}(x)\right) \mathrm{d} x \geq \liminf _{h \rightarrow 0} \int_{\Omega} Q\left(x, \chi_{h}(x) \nabla g_{h}(x)\right) \mathrm{d} x .
$$

Since $\nabla g_{h} \rightarrow \nabla g$ weakly in $L^{2}\left(\Omega ; \mathbb{M}^{n}\right)$ and $\chi_{h} \rightarrow 1$ boundedly in measure (due to (5.6)), we find that

$$
\chi_{h} \nabla g_{h} \rightarrow \nabla g \quad \text { weakly in } L^{2}\left(\Omega ; \mathbb{M}^{n}\right)
$$

and the sequentially weak lower semi-continuity of the quadratic integral functional leads to the desired lower bound estimate (5.5).

\section{Homogenization And LinEARization COMmute}

In this section we prove that the diagram (1.3) in Theorem 1.4 commutes. We have to show that the $\Gamma$-convergence statements

$$
\begin{array}{llll}
(1) & \mathcal{I}^{\varepsilon, h} \stackrel{\Gamma}{\longrightarrow} \mathcal{I}_{\text {lin }}^{\varepsilon} & (2) & \mathcal{I}^{\varepsilon, h} \stackrel{\Gamma}{\longrightarrow} \mathcal{I}_{\text {hom }}^{h} \\
(3) & \mathcal{I}_{\text {lin }}^{\varepsilon} \stackrel{\Gamma}{\longrightarrow} \mathcal{I}^{0} & (4) & \mathcal{I}_{\text {hom }}^{h} \stackrel{\Gamma}{\longrightarrow} \mathcal{I}^{0}
\end{array}
$$

hold w.r.t. strong convergence in $L^{2}\left(\Omega ; \mathbb{R}^{n}\right)$.

First, we discuss the $\Gamma$-limits corresponding to linearization, i.e. (1) and (4). To this end we recall that Proposition 1.6 implies that

$$
\min \left\{\mathcal{I}^{\varepsilon, h}(g), \mathcal{I}_{\text {hom }}^{h}(g)\right\} \geq c\|g\|_{W^{1,2}\left(\Omega ; \mathbb{R}^{n}\right)}^{2}
$$

for all $g \in \mathcal{A}_{\gamma}$ and $h, \varepsilon>0$. As a consequence we see that convergence (1) directly follows by applying Theorem 5.1 to the sequence $\left(\mathcal{I}^{\varepsilon, h}\right)_{h}$.

For the justification of convergence (4), we first apply Theorem 1.1 and see that $W_{\mathrm{hom}}^{(\mathrm{mc})}$ admits a quadratic Taylor expansion. It is well-known that the homogenization $Q_{\mathrm{hom}}^{(1)}$ of a quadratic integrand $Q \in \mathcal{Q}$ is a quadratic form over $\mathbb{R}^{n}$, and 
therefore satisfies the condition of Theorem [5.1 Moreover, Proposition 1.6 proves that the sequence $\left(\mathcal{I}_{\text {hom }}^{h}\right)$ is equi-coercive and (4) follows by applying Theorem 5.1

The $\Gamma$-limits corresponding to homogenization, i.e. (2) and (3), follow by standard results. More precisely, convergence (3) is a classical result in convex homogenization (cf. e.g. 10, 18 and note that these results can be restated in the language of $\Gamma$-convergence). The non-convex homogenization, convergence (2), can be justified by the following result:

Theorem 6.1 (A. Braides [4, S. Müller [16]). Let $\Omega$ be a Lipschitz domain in $\mathbb{R}^{n}$. Suppose that $W: \mathbb{R}^{n} \times \mathbb{M}^{n} \rightarrow \mathbb{R}$ is a Carathéodory function that is $Y$-periodic in its first variable and that satisfies the growth and coercivity condition (W1) with $p \in(1, \infty)$. We consider the functionals

$$
\mathcal{E}^{\varepsilon}(u):= \begin{cases}\int_{\Omega} W(x / \varepsilon, \nabla u(x)) \mathrm{d} x & \text { if } u \in W^{1, p}\left(\Omega ; \mathbb{R}^{n}\right) \\ +\infty & \text { else },\end{cases}
$$

and

$$
\mathcal{E}_{\mathrm{hom}}(u):= \begin{cases}\int_{\Omega} W_{\mathrm{hom}}^{(\mathrm{mc})}(\nabla u(x)) \mathrm{d} x & \text { if } u \in W^{1, p}\left(\Omega ; \mathbb{R}^{n}\right) \\ +\infty & \text { else. }\end{cases}
$$

Then $\left(\mathcal{E}^{\varepsilon}\right) \Gamma$-converges to $\mathcal{E}_{\mathrm{hom}}$ with respect to strong convergence in $L^{p}\left(\Omega ; \mathbb{R}^{n}\right)$ as $\varepsilon \rightarrow 0$.

The previous theorem can be adapted to the boundary conditions considered in diagram (1.3). More precisely, one can show that

for every $u \in W^{1, p}\left(\Omega ; \mathbb{R}^{n}\right)$ there is a sequence $\left(u_{\varepsilon}\right)$ such that $u_{\varepsilon} \rightarrow u$ strongly in $L^{p}\left(\Omega ; \mathbb{R}^{n}\right), u_{\varepsilon}=u$ on $\partial \Omega$ and $\lim _{\varepsilon \rightarrow 0} \mathcal{E}^{\varepsilon}\left(u_{\varepsilon}\right)=\mathcal{E}_{\text {hom }}(u)$.

This can be seen by a standard gluing argument (cf. e.g. [16, 5]). In particular, we can apply this modification in the case $p \geq 2$ and to limiting deformations $u \in W^{1,2}\left(\Omega ; \mathbb{R}^{n}\right)$ satisfying $u(x)=x$ on $\gamma$. Hence, the theorem implies that $\left(\mathcal{I}^{\varepsilon, h}\right)_{\varepsilon}$ $\Gamma$-converges to $\mathcal{I}_{\text {hom }}^{h}$ with respect to the strong topology in $L^{2}\left(\Omega ; \mathbb{R}^{n}\right)$.

In conclusion we see that both paths (1), (3) and (2), (4) lead to the same limiting functional $\mathcal{I}^{0}$ and the diagram commutes.

\section{Failure of commutativity}

In this section we present two examples illustrating that homogenization and linearization cannot be interchanged in general. In the first example we consider energy densities of class $\mathcal{W}(a, p)$ and show that the commutativity might fail if the expansion is not centered at identity. Secondly, we elaborate on the importance of condition (W2) and (W3), which imply that the material has a single energy well at $S O(n)$ with quadratic growth. As an example we discuss a perforated composite with a prestressed component and give an indirect argument that homogenization and linearization do not commute at identity - although the homogenized energy density is stress free at identity and satisfies $W_{\mathrm{hom}}^{(\mathrm{mc})}(\mathrm{Id}+G)=O\left(|G|^{2}\right)$. 
7.1. Counterexample I: Non-commutativity for expansions away from $S O(n)$. In this section we argue that in general the commutability of homogenization and linearization does not hold for expansions centered at $F \notin S O(n)$. Roughly speaking, the reason is the following: If $F \notin S O(n)$ we may find a nonlinear material $W \in \mathcal{W}(a, p)$ with $W_{\text {hom }}^{(\mathrm{mc})}(F)<W_{\text {hom }}^{(1)}(F)$, which means that long-wave oscillations with period $k \varepsilon, k \geq 2$, lead to limiting energies that are lower than those obtained by "one-cell" oscillations with period $\varepsilon$. On the other side, the linearized energy and its homogenization is always quadratic, and due to the non-degeneracy of the material stable in a neighborhood of $S O(n)$. Thus, low energy states related to long-wave oscillations are ignored by the homogenization of the linearized energy; and therefore homogenization and linearization do not commute for $F \notin S O(n)$ in general.

In the rest of this section we present an implicit, but rigorous formulation of the idea above by varying an example introduced by the first author in 16. Let $W_{0}: \mathbb{M}^{2} \rightarrow[0, \infty)$ be a frame indifferent integrand of class $C^{3}$. We suppose that $W_{0}$ satisfies the local Lipschitz- and growth condition (W1) with $p=2$, and

$$
\frac{1}{c} \operatorname{dist}^{2}(F, S O(2)) \leq W_{0}(F) \leq c \operatorname{dist}^{2}(F, S O(2)) \quad \text { for all } F \in \mathbb{M}^{2} .
$$

We consider the periodic stored energy function

$$
W(y, F):=(\chi(y)+\alpha(1-\chi(y))) W_{0}(F),
$$

where $\alpha$ is a small positive parameter and $\chi$ denotes the indicator function of the periodic pattern

$$
P:=\left\{\left(x_{1}, x_{2}\right) \in \mathbb{R}^{2}: \exists k \in \mathbb{Z} \text { such that } x_{2} \in\left[k, k+\frac{1}{2}\right)\right\}
$$

Thus, $W$ describes a composite material with a layered microstructure consisting of a stiff component on $P$ and a soft matrix with stiffness $\alpha$.

As a main result we prove a necessary condition for the property that linearization and homogenization commute for all expansions in an neighborhood of Id:

Proposition 7.1. Let $U$ be an open neighborhood of Id. Suppose that for each expansion at $F \in U$ homogenization and linearization commute in the following sense: There exists $\sigma_{F} \in \mathbb{M}^{2}$ such that

$$
\forall G \in \mathbb{M}^{2}: \quad W_{\mathrm{hom}}^{(\mathrm{mc})}(F+G)=W_{\mathrm{hom}}^{(\mathrm{mc})}(F)+\left\langle\sigma_{F}, G\right\rangle+Q_{\mathrm{hom}}^{F}(G)+o\left(|G|^{2}\right)
$$

where $Q_{\mathrm{hom}}^{F}$ denotes the homogenization of the quadratic integrand

$$
Q^{F}(y, G):=\frac{1}{2} D^{2} W(y, F)(G, G) .
$$

Then

$$
\alpha \geq c\left(W_{0}, U\right)
$$

for a positive constant $c\left(W_{0}, U\right)>0$ that only depends on $W_{0}$ and the neighborhood $U$.

Remark 7.2. Theorem 1.1 implies that the expansion holds for $F=\operatorname{Id}$ with $\sigma_{F}=0$.

Remark 7.3. The result can be read as follows: For any open neighborhood $U$ of Id we can find a material (e.g. by choosing $\alpha$ sufficiently small) such that homogenization and linearization do not commute for all expansions centered in $U$. 
The result follows from a slightly stronger statement that we prove below. In particular, it is sufficient to study the response of the homogenized material to compressions of the form

$$
x \mapsto F_{\delta} x, \quad F_{\delta}:=\operatorname{Id}-\delta\left(e_{1} \otimes e_{1}\right), \quad \delta \geq 0
$$

For future reference we set

$$
Q^{\delta}(y, G):=\frac{1}{2} D^{2} W\left(y, F_{\delta}\right)(G, G)
$$

and let $Q_{\text {hom }}^{\delta}$ denote its homogenization.

In [16] it was shown that if $\delta>0$ and $\alpha$ is sufficiently small, the stiff part of the material, which resembles an ensemble of aligned rods, starts to buckle and allows to bound the limiting energy linearly in $\alpha$. In contrast to this, we prove that the non-degeneracy of $W$ implies that $Q_{\text {hom }}^{\delta}$ is stable for small $\delta$. Both observations can be quantified as follows:

Lemma 7.4. There exist positive constants $\delta_{0}$ and $c_{0}$ (depending only on $W_{0}$ ) with the following properties: For all $0 \leq \delta \leq \delta_{0}$ and $\alpha>0$ we have

$$
\begin{aligned}
Q_{\mathrm{hom}}^{\delta}\left(e_{1} \otimes e_{1}\right) & \geq \frac{1}{c_{0}}, \\
W_{\mathrm{hom}}^{(\mathrm{mc})}\left(F_{\delta}\right) & \leq c_{0} \alpha .
\end{aligned}
$$

We postpone the proof to the end of this section and present a quite immediate consequence of the lemma that already implies the validity of Proposition 7.1 .

Lemma 7.5. Define the map

$$
f: \mathbb{R} \rightarrow \mathbb{R}, \quad f(\delta):=W_{\mathrm{hom}}^{(\mathrm{mc})}\left(F_{\delta}\right) .
$$

Then for almost every $\delta \in\left[0, \delta_{0}\right]$ the map $f$ admits a quadratic Taylor expansion of the form

$$
f(\delta+\lambda)=f(\delta)+\sigma_{\delta} \lambda+q_{\delta} \lambda^{2}+o\left(\lambda^{2}\right)
$$

for suitable numbers $\sigma_{\delta} \in \mathbb{R}$ and $q_{\delta} \geq 0$.

(1) If $\delta=0$, the expansion (7.4) is valid for

$$
\sigma_{0}=0 \quad \text { and } \quad q_{0}=Q_{\mathrm{hom}}^{0}\left(e_{1} \otimes e_{1}\right),
$$

i.e. linearization and homogenization commute at $F_{0}=\mathrm{Id}$.

(2) Suppose that linearization and homogenization commute at $F_{\delta}$ for all a.e. $\delta \in\left[0, \delta_{0}\right]$, i.e.

$$
q_{\delta}=Q_{\mathrm{hom}}^{\delta}\left(e_{1} \otimes e_{1}\right) \quad \text { for a.e. } \delta \in\left[0, \delta_{0}\right] .
$$

Then

$$
\alpha \geq \frac{\delta_{0}^{2}}{2 c_{0}^{2}} .
$$

Remark 7.6. In the lemma above the constants $\delta_{0}$ and $c_{0}$ only depend on $W_{0}$. Thus, if the soft matrix of the composite material is sufficiently weak, i.e. $\alpha \ll 1$, statement (2) suggests that homogenization and linearization may only commute in a very small neighborhood of Id. 
Proof of Lemma 7.5. By Lemma 1.3 the map $W_{\mathrm{hom}}^{(\mathrm{mc})}: \mathbb{M}^{2} \rightarrow \mathbb{R}$ is continuous and quasiconvex, and therefore rank-one convex. Because $F_{\delta}-F_{\lambda}$ is always a rank-one matrix, we deduce that $f$ is convex and continuous. Thus, Aleksandrov's Theorem implies that $f$ admits an expansion of the form (7.4) for a.e. $\delta \in\left[0, \delta_{0}\right]$. Statement (1) is a direct consequence of Theorem 1.1 applied to $G=-\lambda\left(e_{1} \otimes e_{1}\right)$.

We prove (2). By assumption we have

$$
f^{\prime \prime}(\delta)=Q_{\mathrm{hom}}^{\delta}\left(e_{1} \otimes e_{1}\right)
$$

for a.e. $\delta$. We apply (7.2) from Lemma 7.4 and find that $f^{\prime \prime} \geq c_{0}^{-1}$ almost everywhere. Thus, the map

$$
g(\delta):=f(\delta)-\frac{1}{2 c_{0}} \delta^{2}
$$

is convex and by part (1) we have $g^{\prime}(0)=f^{\prime}(0)=0$. Thus $g$ attains its minimum at 0 with $g(0)=0$. By (7.3) we get

$$
\frac{1}{2 c_{0}} \delta^{2} \leq f(\delta) \leq c_{0} \alpha \quad \text { for all } \delta \in\left[0, \delta_{0}\right]
$$

and the estimate for $\alpha$ follows.

Proof of Lemma 7.4. In the sequel $c^{\prime}, c^{\prime \prime}$ denote positive constants that may change from line to line, but can be chosen only depending on $\delta_{0}$ and $W_{0}$.

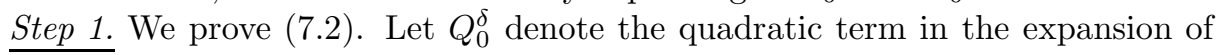
$W_{0} \overline{\text { at } F_{\delta}} \in \mathbb{M}^{2}$, i.e.

$$
Q_{0}^{\delta}(G):=\frac{1}{2} D^{2} W_{0}\left(F_{\delta}\right)(G, G) .
$$

Because $F_{0}=\mathrm{Id}$ and $W_{0}$ is frame-indifferent and non-degenerate, the estimate

$$
Q_{0}^{0}(G) \geq \frac{1}{c^{\prime}}|\operatorname{sym} G|^{2}
$$

holds for all $G \in \mathbb{M}^{2}$. The map $W_{0}$ is of class $C^{3}$, and therefore a standard perturbation argument shows that

$$
Q_{0}^{\delta}(G) \geq \frac{1}{c^{\prime}}|\operatorname{sym} G|^{2}-c^{\prime \prime} \delta|\operatorname{skw} G|^{2}
$$

for all $\delta \in\left[0, \delta_{0}\right]$ where $\delta_{0}$ denotes a small positive constant that only depends on $W_{0}$.

Let $Y_{0}:=[0,1) \times\left[0, \frac{1}{2}\right)$ denote the reference domain of the stiff component and consider the function space

$$
V:=\left\{\psi \in W^{1,2}\left(Y_{0} ; \mathbb{R}^{2}\right): \psi\left(0, x_{2}\right)=\psi\left(1, x_{2}\right), \int_{Y_{0}} \psi(x) \mathrm{d} x=0\right\} .
$$

Note that for all $\psi \in V$ a Korn inequality of the form

$$
\int_{Y_{0}}|\operatorname{skw} \nabla \psi|^{2} \mathrm{~d} y \leq c^{\prime} \int_{Y_{0}}|\operatorname{sym} \nabla \psi|^{2} \mathrm{~d} y
$$

holds. As a consequence we deduce that

$$
m\left(\delta_{0}\right):=\inf _{\delta \in\left(0, \delta_{0}\right)} \inf _{\psi \in V} \int_{Y_{0}} Q_{0}^{\delta}\left(e_{1} \otimes e_{1}+\nabla \psi(x)\right) \mathrm{d} y>0
$$

provided $\delta_{0}$ is sufficiently small. 
Next, we are going to show that the estimate above yields a lower bound for $Q_{\text {hom }}^{\delta}(G)$ : Let $\delta \in\left[0, \delta_{0}\right]$ and $\psi \in W_{\text {per }}^{1,2}\left(Y ; \mathbb{R}^{2}\right)$. Then

$$
\int_{Y} Q^{\delta}\left(y,\left(e_{1} \otimes e_{1}\right)+\nabla \psi\right) \mathrm{d} y \geq \int_{Y_{0}} Q_{0}^{\delta}\left(\left(e_{1} \otimes e_{1}\right)+\nabla \psi\right) \mathrm{d} y \geq m\left(\delta_{0}\right)>0
$$

and (17.2) is valid for all $c_{0} \geq m\left(\delta_{0}\right)^{-1}$.

Step 2. We prove (7.3) by constructing a sequence $\left(u_{k}\right)_{k \in \mathbb{N}}$ such that

$$
\liminf _{k \rightarrow \infty} \int_{Y} W\left(k x, \nabla u_{k}(x)\right) \mathrm{d} x \leq c_{0} \alpha
$$

and $u_{k} \rightarrow F_{\delta} x+\varphi(x)$ weakly in $W^{1,2}\left(Y ; \mathbb{R}^{2}\right)$ with $\varphi \in W_{\text {per }}^{1,2}\left(Y ; \mathbb{R}^{2}\right)$. Because the functional

$$
W^{1,2}\left(Y ; \mathbb{R}^{2}\right) \ni u \mapsto \int_{Y} W(k x, \nabla u(x)) \mathrm{d} x
$$

$\Gamma$-converges in the strong topology of $L^{2}\left(Y ; \mathbb{R}^{2}\right)$ to

$$
W^{1,2}\left(Y ; \mathbb{R}^{2}\right) \ni u \mapsto \int_{Y} W_{\mathrm{hom}}^{(\mathrm{mc})}(\nabla u(x)) \mathrm{d} x
$$

as $k \rightarrow \infty$, (7.5) implies that

$$
\int_{Y} W_{\mathrm{hom}}^{(\mathrm{mc})}\left(F_{\delta}+\nabla \varphi(x)\right) \mathrm{d} x \leq \liminf _{k \rightarrow \infty} \int_{Y} W\left(k x, \nabla u_{k}(x)\right) \mathrm{d} x \leq c_{0} \alpha .
$$

The homogenized integrand $W_{\mathrm{hom}}^{(\mathrm{mc})}$ is quasiconvex, and therefore the left hand side is bounded from below by $W_{\mathrm{hom}}^{(\mathrm{mc})}\left(F_{\delta}\right)$.

It remains to construct the sequence $\left(u_{k}\right)$. We follow the idea in [16]: The microstructure of the material can be regarded as an ensemble of thin rods, aligned to the $e_{1}$-direction and embedded in a soft matrix. The subsequent construction realizes the compression $x \mapsto F_{\delta} x$ (more precisely, a periodic variation) by bending each rod. In this way, locally the deformation in the stiff component is close to a rigid motion, while large strains only evolve in the soft matrix.

For the precise construction, let $v_{\delta} \in C^{\infty}\left([0,1] ; \mathbb{R}^{2}\right)$ denote a curve, parametrized by arc-length and rendering an arc with length 1 connecting the points $(0,0)$ and $(1-\delta, 0)$, i.e.

$$
v_{\delta}(0)=(0,0), \quad v_{\delta}(1)=(1-\delta, 0), \quad\left|v_{\delta}^{\prime}\left(x_{1}\right)\right|=1, \quad\left|v_{\delta}^{\prime \prime}\left(x_{1}\right)\right|=\kappa_{\delta}
$$

for some $\kappa_{\delta}>0$. Let $n_{\delta} \in C^{\infty}\left([0,1] ; \mathbb{R}^{2}\right)$ denote a normal field associated to the curve, so that the map $x_{1} \mapsto R_{\delta}\left(x_{1}\right):=v_{\delta}^{\prime}\left(x_{1}\right) \otimes e_{1}+n_{\delta}\left(x_{1}\right) \otimes e_{2}$ only takes values in $S O(2)$.

For $x \in Y$ define

$$
u_{k}(x):=\left\{\begin{array}{lll}
x_{2} e_{2}+v_{\delta}\left(x_{1}\right)+\left(x_{2}-\frac{i}{k}\right)\left(n_{\delta}\left(x_{1}\right)-e_{2}\right) & \text { if } \quad x_{2} \in\left[\frac{i}{k}, \frac{2 i+1}{2 k}\right) \\
& \text { for some } i \in \mathbb{N}_{0}, \\
x_{2} e_{2}+v_{\delta}\left(x_{1}\right)+\left(\frac{i+1}{k}-x_{2}\right)\left(n_{\delta}\left(x_{1}\right)-e_{2}\right) & \text { if } \quad x_{2} \in\left[\frac{2 i+1}{2 k}, \frac{i+1}{k}\right) \\
& \text { for some } i \in \mathbb{N}_{0} .
\end{array}\right.
$$

By construction we have $u_{k} \in W^{1,2}\left(Y ; \mathbb{R}^{2}\right)$ and $u_{k} \rightarrow v_{\delta}+x_{2} e_{2}$ uniformly. The sequence $\left(u_{k}\right)$ is bounded in $W^{1,2}\left(Y ; \mathbb{R}^{2}\right)$ and therefore

$$
u_{k} \rightarrow F_{\delta} x+\varphi(x) \quad \text { weakly in } W^{1,2}\left(Y ; \mathbb{R}^{2}\right)
$$


where $\varphi(x):=v_{\delta}\left(x_{1}\right)+x_{2} e_{2}-F_{\delta} x$. Furthermore, it is easy to check that $\varphi$ can be identified with a map in $W_{\text {per }}^{1,2}\left(Y ; \mathbb{R}^{2}\right)$.

$\nabla u_{n}$ is close to a rotation on the strong part of the material. More precisely, we have

$$
\operatorname{dist}^{2}\left(\nabla u_{k}(x), S O(2)\right) \leq\left(\frac{\kappa_{\delta}^{2}}{4}\right) k^{-2} \quad \text { for all } x_{2} \in\left[\frac{i}{k}, \frac{2 i+1}{2 k}\right),
$$

while on the soft part the deformation is at least bounded, i.e.

$$
\left|\nabla u_{k}(x)\right|^{2} \leq c^{\prime} \quad \text { for all } x_{2} \in\left[\frac{2 i+1}{2 k}, \frac{i+1}{k}\right) .
$$

Thus, by using the definition of $W$ and the growth condition (7.1), we see that

$$
\begin{array}{r}
\int_{Y} W\left(k x, \nabla u_{k}(x)\right) \mathrm{d} x \leq c^{\prime} \sum_{i=0}^{k-1}\left(\int_{0}^{1} \int_{i / k}^{(2 i+1) / 2 k} \operatorname{dist}^{2}\left(\nabla u_{k}, S O(2)\right) \mathrm{d} x_{2}\right. \\
\left.+\alpha \int_{0}^{1} \int_{(2 i+1) / 2 k}^{(i+1) / k}\left|\nabla u_{k}\right|^{2}+2 \mathrm{~d} x_{2} \mathrm{~d} x_{1}\right) \\
\leq c^{\prime}\left(k^{-2}+\alpha\right)
\end{array}
$$

and (7.6) follows as $k \rightarrow \infty$.

7.2. Counterexample II: Non-commutativity at identity for a perforated, prestressed composite material. In this section we construct an example in dimension 3 for which the commutativity of linearization and homogenization at identity fails, although the identity is a natural state of the homogenized material. The material under consideration is a composite with a prestressed component that violates property (W2). Furthermore, the material is perforated and the nondegeneracy condition (W3) is violated in $x_{3}$-direction.

A commutativity statement in the form of Theorem 1.1 comparing the homogenized and the spatially heterogeneous energy density, is not suited for a prestressed material due to the lack of a stress free reference configuration. Therefore, we introduce a weaker property that is motivated by the following observation:

Corollary 7.7. Let $W \in \mathcal{W}(a, p)$. For $k \in \mathbb{N}$ let

$$
W_{\text {hom }}^{(k)}(F):=\inf \left\{\frac{1}{k^{n}} \int_{k Y} W(y, F+\nabla \varphi(y)) \mathrm{d} y: \varphi \in W_{\text {per }}^{1,2}\left(k Y ; \mathbb{R}^{n}\right)\right\}
$$

denote the $k$-cell homogenization of $W$. Then

$$
\lim _{h \downarrow 0} \frac{1}{h^{2}} W_{\mathrm{hom}}^{(k)}(\mathrm{Id}+h G)=\lim _{h \downarrow 0} \frac{1}{h^{2}} W_{\mathrm{hom}}^{(\mathrm{mc})}(\mathrm{Id}+h G) .
$$

for all $k \in \mathbb{N}$ and all $G \in \mathbb{M}^{n}$.

Proof. The statement is implicit in the proof of Theorem 1.1. Indeed, in Step 1 we showed that $\limsup \frac{1}{h^{2}} W_{\text {hom }}^{(1)}(\operatorname{Id}+h G) \leq Q_{\text {hom }}^{(1)}(G)$, while in Step 2 we proved the inequality $\liminf _{h \downarrow 0} \frac{1}{h^{2}} W_{\mathrm{hom}}^{(\mathrm{mc})}(\operatorname{Id}+h G) \geq Q_{\mathrm{hom}}^{(1)}(G)$. Since $W_{\mathrm{hom}}^{(\mathrm{mc})} \leq W_{\mathrm{hom}}^{(k)} \leq W_{\mathrm{hom}}^{(1)}$ we can draw the conclusion. 
The corollary indicates that in situations when commutativity holds, close to identity relaxation over a single cell is sufficient for homogenization. Proposition 7.1 shows that if we replace Id by a matrix $F \notin S O(n)$, identity (7.7) fails for some materials of class $\mathcal{W}(a, p)$. This suggests to introduce the following property: We say that homogenization and linearization in direction $G \in \mathbb{M}^{n}$ commute at Id, if

$$
\begin{aligned}
& \text { for all } k \in \mathbb{N} \text { there exist } \sigma^{(k)}, \sigma \in \mathbb{M}^{n} \text { such that } \\
& \left.\qquad \begin{array}{rl}
\lim _{h \downarrow 0} \frac{1}{h^{2}}\left(W_{\text {hom }}^{(k)}(\operatorname{Id}+h G)-W_{\text {hom }}^{(k)}(\mathrm{Id})-h\left\langle\sigma^{(k)}, G\right\rangle\right) \\
\quad=\lim _{h \downarrow 0} \frac{1}{h^{2}}\left(W_{\mathrm{hom}}^{(\mathrm{mc})}(\mathrm{Id}+h G)-W_{\mathrm{hom}}^{(\mathrm{mc})}(\mathrm{Id})-h\langle\sigma, G\rangle\right)
\end{array}\right\}
\end{aligned}
$$

Note that we distinguish between the directions $+G$ and $-G$. By Corollary 7.7 materials of class $\mathcal{W}(a, p)$ satisfy $\left(C_{G}\right.$ for all $G \in \mathbb{M}^{n}$.

In the following, we present an example in dimension $n=3$ that violates $\left(C_{G}\right)$. Let us first describe the geometry and the energy density of the composite. The composite's geometry is given by two subsets of the reference cell $Y:=[0,1)^{3}$ :

$$
\begin{gathered}
Y_{0}:=[0,1)^{2} \times\left[0, \frac{1}{2}\right) \quad \text { and } \quad Y_{\rho}:=[0,1) \times B_{\rho}, \\
\text { where } B_{\rho}:=\left\{\left(y_{2}, y_{3}\right):\left(y_{2}-\frac{1}{2}\right)^{2}+\left(y_{3}-\frac{3}{4}\right)^{2} \leq \rho^{2}\right\}, \quad 0<\rho \ll 1 .
\end{gathered}
$$

The set $Y_{\rho}$ is a cylinder with center line $\left\{\left(y_{1}, \frac{1}{2}, \frac{3}{4}\right): y_{1} \in[0,1)\right\}$ and small radius $\rho$. Let $W_{0}$ be of class $\mathcal{W}(a, 2)$. We suppose that $W_{0}$ is frame-indifferent, i.e. $W_{0}(R F)=W_{0}(F)$ for all matrices $F \in \mathbb{M}^{3}$ and rotations $R \in S O(3)$. Set $S:=$ $\left(\operatorname{Id}+s\left(e_{1} \otimes e_{1}\right)\right)^{-1}$ with $0<s<\frac{1}{2}$. We denote by $W: \mathbb{R}^{3} \times \mathbb{M}^{3} \rightarrow[0, \infty)$ the $Y$-periodic energy density

$$
W(y, F):= \begin{cases}W_{0}(F) & \text { if } y \in Y_{0} \\ W_{0}(F S) & \text { if } y \in Y_{\rho} \\ 0 & \text { if } y \in Y \backslash\left(Y_{0} \cup Y_{\rho}\right) .\end{cases}
$$

Clearly, for the material occupying $Y_{0}$ every rotation is a natural state, while on $Y_{\rho}$ the material is stress free only for matrices $F=R S^{-1}$ with $R \in S O(3)$.

Lemma 7.8. The following properties hold:

a) $W$ is frame-indifferent, has quadratic growth at infinity and is non-degenerate in $x_{1}$ - and $x_{2}$-direction in the sense that

$$
\begin{aligned}
& \int_{Y} W(y, F+\nabla \psi(y)) \mathrm{d} y \geq c^{\prime} \operatorname{dist}^{2}(F, S O(3)) \\
& \quad \text { for all } \psi \in W_{\mathrm{per}}^{1,2}\left(Y ; \mathbb{R}^{3}\right) \text { with } \int_{0}^{1} \int_{0}^{1} \partial_{3} \psi \mathrm{d} y_{1} \mathrm{~d} y_{2}=0 .
\end{aligned}
$$

b) For all $k \in \mathbb{N}$ we have $\inf _{F \in \mathbb{M}^{3}} W_{\text {hom }}^{(k)}(F)>0$.

c) (Natural state).

$$
W_{\mathrm{hom}}^{(\mathrm{mc})}(\mathrm{Id})=0=\min _{F \in \mathbb{M}^{3}} W_{\mathrm{hom}}^{(\mathrm{mc})}(F) .
$$

d) (Expansion at Id). For all $G \in \mathbb{M}^{3}$ we have

$$
\limsup _{h \rightarrow 0} \frac{1}{h^{2}} W_{\text {hom }}^{(\mathrm{mc})}(\operatorname{Id}+h G)<\infty .
$$


e) (Failure of $\left(C_{G}\right)$ ). There exists $G \in \mathbb{M}^{3}$ such that for all $k \in \mathbb{N}$ and all $\sigma^{(k)} \in \mathbb{M}^{3}$ with

$$
\limsup _{h \downarrow 0} \frac{1}{h^{2}}\left|W_{\text {hom }}^{(k)}(\mathrm{Id}+h G)-W_{\text {hom }}^{(k)}(\mathrm{Id})-h\left\langle\sigma^{(k)}, G\right\rangle\right|<\infty
$$

we have

$$
\begin{aligned}
\liminf _{h \downarrow 0} \frac{1}{h^{2}}\left(W_{\text {hom }}^{(k)}(\operatorname{Id}+h G)-W_{\text {hom }}^{(k)}(\mathrm{Id})-\right. & \left.h\left\langle\sigma^{(k)}, G\right\rangle\right) \\
& >0=\lim _{h \downarrow 0} \frac{1}{h^{2}} W_{\text {hom }}^{(\mathrm{mc})}(\mathrm{Id}+h G) .
\end{aligned}
$$

In a nutshell, the idea behind the construction is the following: Close to identity and for finite $k$, the prestressed component contributes a positive amount of energy and yields a shift of the natural state to some $F \notin S O(3)$. When $k$ increases, the aspect ratio (thickness / length) of the cylindrical component and (as a consequence) the contributed elastic energy decreases. We show that in the limit $k \rightarrow \infty$ the energy contribution of the $Y_{\rho}$-component vanishes. Furthermore, we argue that $W_{\mathrm{hom}}^{(\mathrm{mc})}$ vanishes for short maps.

Proof of Lemma 7.8. Let us introduce the functionals

$$
\begin{array}{rlrl}
I^{(k)}(\varphi ; F) & \left.:=\frac{1}{k^{3}} \int_{k Y} W(y, F+\nabla \varphi)\right) \mathrm{d} y, & & \\
I_{0}^{(k)}(\varphi ; F) & :=\frac{1}{k^{2}} \int_{Z_{0}^{(k)}} W_{0}(F+\nabla \varphi) \mathrm{d} y, & & Z_{0}^{(k)}:=(0, k)^{2} \times\left(0, \frac{1}{2}\right), \\
I_{\rho}^{(k)}(\varphi ; F):=\frac{1}{k} \int_{Z_{\rho}^{(k)}} W_{0}((F+\nabla \varphi) S) \mathrm{d} y, & Z_{\rho}^{(k)}:=(0, k) \times B_{\rho} .
\end{array}
$$

and the function spaces

$$
\begin{aligned}
& X^{(k)}:=W_{\text {per }}^{1, p}\left(k Y ; \mathbb{R}^{3}\right), \\
& X_{0}^{(k)}:=\left\{\left.\varphi\right|_{Z_{0}^{(k)}}: \varphi \in X^{(k)}\right\}, \\
& X_{\rho}^{(k)}:=\left\{\left.\varphi\right|_{Z_{\rho}^{(k)}}: \psi \in X^{(k)}\right\} .
\end{aligned}
$$

Note that by definition we have $W_{\text {hom }}^{(k)}(F)=\inf _{\varphi \in X^{(k)}} I^{(k)}(\varphi ; F)$.

The proof is divided in several steps. In Step 1 we argue that since the components $Y_{0}$ and $Y_{\rho}$ are separated, we can split $I^{(k)}$ into $I_{0}^{(k)}$ and $I_{\rho}^{(k)}$. In Step 2 we argue that $I_{\rho}^{(k)}(\cdot ; F)$ is positive for $F$ close to Id and show that its contribution vanishes as $k \rightarrow \infty$. In Step 3 we analyze the behavior of $I_{0}^{(k)}$. In particular, for the asymptotic behavior $k \rightarrow \infty$ we appeal to a $\Gamma$-convergence result for membranes (see [14, 7]). In Step 4 we draw the conclusion.

$\underline{\text { Step 1. }}$ (Splitting of $\left.I^{(k)}\right)$. We claim that for all $(\varphi, \psi) \in X_{0}^{(k)} \times X_{\rho}^{(k)}$ there exists $\Phi \overline{\in X^{(k)}}$ such that

$$
I^{(k)}(\Phi ; F)=I_{0}^{(k)}(\varphi ; F)+I_{\rho}^{(k)}(\psi ; F)
$$

and

$$
\inf _{\Phi \in X^{(k)}} I^{(k)}(\Phi ; F)=\inf _{\varphi \in X_{0}^{(k)}} I_{0}^{(k)}(\varphi ; F)+\inf _{\psi \in X_{\rho}^{(k)}} I_{\rho}^{(k)}(\psi ; F) .
$$


Statement (7.10) easily follows from a periodicity property of the composite's microstructure. Namely, we can rewrite the subset of the multi-cell $k Y$ occupied by the $Y_{0}$-material as a union of $k$ translations of the set $Z_{0}^{(k)}$; and the subset occupied by the $Y_{\rho}$-material as $k^{2}$ translations of the set $Z_{\rho}^{(k)}$ :

$$
\begin{aligned}
& Y_{0}^{(k)}:=\bigcup_{\xi \in \mathbb{Z}^{3} \cap[0, k)^{3}}\left(\xi+Y_{0}\right)=\bigcup_{i \in \mathbb{Z} \cap[0, k)}\left((0,0, i)+Z_{0}^{(k)}\right), \\
& Y_{\rho}^{(k)}:=\bigcup_{\xi \in \mathbb{Z}^{3} \cap[0, k)^{3}}\left(\xi+Y_{\rho}\right)=\bigcup_{i, j \in \mathbb{Z} \cap[0, k)}\left((0, i, j)+Z_{\rho}^{(k)}\right) .
\end{aligned}
$$

For $y \in Y_{0}^{(k)} \cup Y_{\rho}^{(k)}$ set

$$
\widetilde{\Phi}(y):= \begin{cases}\varphi(y) & \text { if } y \in(0,0, i)+Z_{0}^{(k)} \text { for some } i \in \mathbb{Z} \\ \psi(y) & \text { if } y \in(0, i, j)+Z_{\rho}^{(k)} \text { for some } i, j \in \mathbb{Z}\end{cases}
$$

Since $\overline{Y_{0}^{(k)}} \cap \overline{Y_{\rho}^{(k)}}=\emptyset$ we can extend $\widetilde{\Phi}$ to a $k Y$-periodic map $\Phi \in X^{(k)}$. Since $W(y, \cdot) \equiv 0$ whenever $y \in(k Y) \backslash\left(Y_{0}^{(k)} \cup Y_{\rho}^{(k)}\right)$ identity (7.10) follows. (77.11) follows from (7.10) and the observation that " $\geq$ " trivially holds in (7.11), since the minimization problems on the r. h. s. are less constrained.

Step 2. (Estimates for $\left.I_{\rho}^{(k)}\right)$. Set

$$
m_{\rho}^{(k)}(F):=\inf _{\psi \in X_{\rho}^{(k)}} I_{\rho}^{(k)}(\psi ; F) .
$$

We claim that

$$
\begin{aligned}
& \forall k \in \mathbb{N}, G \in \mathbb{M}^{3}: \liminf _{h \rightarrow 0} m_{\rho}^{(k)}(\operatorname{Id}+h G)>0 \\
& \forall G \in \mathbb{M}^{3}: \limsup _{k \rightarrow \infty} m_{\rho}^{(k)}(\operatorname{Id}+h G)= \begin{cases}0 & \text { if } G e_{1}=0 \\
O\left(h^{2}\right) & \text { else. }\end{cases}
\end{aligned}
$$

Proof of (7.12) by contradiction: Suppose there exists a vanishing sequence of positive numbers $\left(h_{j}\right)_{j \in \mathbb{N}}$ and a sequence $\left(\psi_{j}\right) \subset X_{\rho}^{(k)}$ such that

$$
\liminf _{j \rightarrow \infty} I_{\rho}^{(k)}\left(\psi_{j} ; \operatorname{Id}+h_{j} G\right)=0 .
$$

Consider the deformation $u_{j}(x):=\left(\operatorname{Id}+h_{j} G\right) x+\psi_{j}(x)$ and the mapping $v_{j}(z):=$ $u_{j}(S z)$ defined for $z \in S^{-1} Z_{\rho}^{(k)}$. Since $\nabla v_{j}(z)=\nabla u_{j}(S z) S$, a change of variables yields

$$
\frac{1}{(1+s) k} \int_{S^{-1} Z_{\rho}^{(k)}} W_{0}\left(\nabla v_{j}\right) \mathrm{d} z=\frac{1}{k} \int_{Z_{\rho}^{(k)}} W_{0}\left(\nabla u_{j}(x) S\right) \mathrm{d} x=I_{\rho}^{(k)}\left(\psi_{j} ; \operatorname{Id}+h_{j} G\right) .
$$

Thus, by (17.14) we have (up to a subsequence)

$$
\frac{1}{(1+s) k} \int_{S^{-1} Z_{\rho}^{(k)}} W_{0}\left(\nabla v_{j}\right) \mathrm{d} z \rightarrow 0 \quad(\text { as } j \rightarrow \infty) .
$$

In combination with the non-degeneracy condition (W3) and by appealing to geometric rigidity (see Theorem 3.1) we find (after passing to a further subsequence) that there exists a rotation $R \in S O(3)$ such that

$$
\nabla v_{j} \rightarrow R \quad \text { strongly in } L^{2}\left(S^{-1} Z_{\rho}^{(k)}\right) .
$$


On the other side, the periodicity of $z \mapsto \psi_{j}(S z)$ in its first variable yields the identity

$$
\frac{1}{\left|S^{-1} Z_{\rho}^{(k)}\right|} \int_{S^{-1} Z_{\rho}^{(k)}} \partial_{1} v_{j} \mathrm{~d} z=\left(\operatorname{Id}+h_{j} G\right) S e_{1} .
$$

By (7.16) the left hand side converges to $R e_{1}$, while the right hand side converges to $S e_{1}$; since $\left|S e_{1}\right| \neq 1=\left|R e_{1}\right|$, this is a contradiction.

The argument for (7.13) relies on a bending ansatz for inextensible rods. Let $v_{0}=\left(v_{0}^{1}, v_{0}^{2}, v_{0}^{3}\right)$ denote a smooth, $(1+s)$-periodic curve from $\mathbb{R}$ to $\mathbb{R}^{3}$ with $\left|v_{0}^{\prime}\right| \equiv 1$ and

$$
v_{0}(0)=\left(0, \frac{1}{2}, \frac{3}{4}\right), \quad v_{0}(1+s)=v_{0}(0)+e_{1}, \quad v_{0}^{\prime}(0)=v_{0}^{\prime}(1+s)=e_{1} .
$$

Further, we assume that the curve is parallel to the $\left(x_{1}, x_{2}\right)$-plane, i.e. $v_{0}^{3} \equiv \frac{3}{4}$. Let $t_{0}:=v_{0}^{\prime}$ denote the tangent, $n_{0}:=-t_{0} \wedge e_{3}$ the normal and $\kappa_{0}:=t_{0} \cdot n_{0}^{\prime}$ the signed curvature of the curve. Now, define the scaled curve

$$
v^{(k)}\left(y_{1}\right):=k v_{0}\left(y_{1} / k\right)
$$

and let $t^{(k)}, n^{(k)}$ and $\kappa^{(k)}$ denote the associated tangent, normal and curvature. Note that the curvature scales as

$$
\kappa^{(k)}\left(y_{1}\right)=\frac{1}{k} \kappa_{0}\left(y_{1} / k\right) .
$$

Based on $v^{(k)}$ we construct a 3 d deformation. For $y \in Z_{\rho}^{(k)}$ set

$$
u_{h}^{(k)}(y):=v^{(k)}\left((1+s) y_{1}\right)+\left(y_{2}-\frac{1}{2}\right) n^{(k)}\left((1+s) y_{1}\right)+\left(y_{3}-\frac{3}{4}\right) e_{3}+h G e_{1} y_{1} .
$$

By construction the map $\psi(y):=u_{h}^{(k)}(y)-(\operatorname{Id}+h G) y$ belongs to $X_{\rho}^{(k)}$. Thus, by definition of $m_{\rho}^{(k)}(\mathrm{Id}+h G)$ we have

$$
m_{\rho}^{(k)}(\mathrm{Id}+h G) \leq \frac{1}{k} \int_{Z_{\rho}^{(k)}} W_{0}\left(\nabla u_{h}^{(k)}(y) S\right) \mathrm{d} y .
$$

A direct computation shows that

$$
\nabla u_{h}^{(k)}=\left(t^{(k)}\left|n^{(k)}\right| e_{3}\right) S^{-1}+\left(y_{2}-\frac{1}{2}\right)\left(\left(n^{(k)}\right)^{\prime} \otimes e_{1}\right) S^{-1}+h G\left(e_{1} \otimes e_{1}\right) .
$$

Multiplication with the rotation $R^{(k)}:=\left(t^{(k)}\left|n^{(k)}\right| e_{3}\right)$ and a short calculation yields the identity

$$
\left(R^{(k)}\right)^{\mathrm{T}} \nabla u_{h}^{(k)} S=\left(\mathrm{Id}+\frac{1}{k} A^{(k)}+h B^{(k)}\right)
$$

with $A^{(k)}(y):=k \kappa^{(k)}\left(y_{1}\right)\left(y_{2}-\frac{1}{2}\right)\left(e_{1} \otimes e_{1}\right)$ and $B^{(k)}:=\left(R^{(k)}\right)^{\mathrm{T}} G\left(e_{1} \otimes e_{1}\right) S$. Since $\left|y_{2}-\frac{1}{2}\right| \leq \rho$ and by appealing to (7.18) and the smoothness of $v_{0}$, we find that

$$
\max _{y \in Z_{\rho}^{(k)}}\left|A^{(k)}(y)\right| \leq c \quad \text { and } \quad\left|B^{(k)}\right| \leq c\left|G e_{1}\right|
$$

where $c$ only depends on the radius $\rho$ and the curve $v_{0}$. Hence, for small $h$ and large $k$ the matrix $\left(R^{(k)}\right)^{\mathrm{T}} \nabla u_{h}^{(k)}(y) S$ is uniformly close to Id and we can appeal to condition (W4) (and the frame indifference of $W_{0}$ ) to justify the following expansion: For $\frac{1}{k} \leq h$ and $h$ sufficiently small there holds (uniformly in $y \in Z_{\rho}^{(k)}$ )

$$
\begin{aligned}
W_{0}\left(\nabla u_{h}^{(k)} S\right) & =W_{0}\left(\left(R^{(k)}\right)^{\mathrm{T}} \nabla u_{h}^{(k)} S\right)=W_{0}\left(\mathrm{Id}+k^{-1} A^{(k)}+h B^{(k)}\right) \\
& =Q_{0}\left(k^{-1} A^{(k)}+h B^{(k)}\right)+o\left(h^{2}\right)
\end{aligned}
$$


where $Q_{0}$ denotes the quadratic form associated with the expansion of $W_{0}$ at identity. Since $\int_{B_{\rho}}\left(y_{2}-\frac{1}{2}\right) \mathrm{d}\left(y_{2}, y_{3}\right)=0$ by symmetry, the components of $A^{(k)}$ and $B^{(k)}$ are orthogonal in $L^{2}\left(B_{\rho}\right)$ and we get

$$
\int_{B_{\rho}} Q_{0}\left(k^{-1} A^{(k)}+h B^{(k)}\right) \mathrm{d}\left(y_{2}, y_{3}\right)=\frac{1}{k^{2}} \int_{B_{\rho}} Q_{0}\left(A^{(k)}\right) \mathrm{d}\left(y_{2}, y_{3}\right)+h^{2}\left|B_{\rho}\right| Q_{0}\left(B^{(k)}\right) .
$$

In conclusion, we have

$$
\limsup _{k \rightarrow \infty} \frac{1}{k} \int_{0}^{k} \int_{B_{\rho}} W_{0}\left(\nabla u_{h}^{(k)} S\right) \mathrm{d} y \leq \begin{cases}0 & \text { if } G e_{1}=0 \\ 2\left|B_{\rho}\right| h^{2} \max _{B} Q_{0}(B)+o\left(h^{2}\right) & \text { else }\end{cases}
$$

where the maximum is taken over the set of all cluster points of the bounded sequence $\left(B^{(k)}\right) \subset \mathbb{M}^{3}$. In combination with (7.19) this implies (7.13).

Step 3. (Properties of $\left.I_{0}^{(k)}\right)$. Set

$$
m_{0}^{(k)}(F):=\inf _{\varphi \in X_{0}^{(k)}} I_{0}^{(k)}(\varphi ; F) .
$$

Let $\mathcal{G}:=\left\{G=-g\left(e_{2} \otimes e_{2}\right): g \in(0,1)\right\}$. We claim that

$$
\begin{gathered}
\forall G \in \mathbb{M}^{3}, k \in \mathbb{N}: \limsup _{h \rightarrow 0} \frac{1}{h^{2}} m_{0}^{(k)}(\operatorname{Id}+h G) \leq \frac{1}{2} Q_{0}(G), \\
\forall G \in \mathcal{G}, k \in \mathbb{N}: \liminf _{h \downarrow 0} \frac{m_{0}^{(k)}(\operatorname{Id}+h G)}{h^{2}}>0, \\
\forall G \in \mathcal{G}: \limsup _{h \downarrow 0} \limsup _{k \rightarrow \infty} \frac{m_{0}^{(k)}(\operatorname{Id}+h G)}{h^{2}}=0 .
\end{gathered}
$$

Argument for (7.20): By definition of $m_{0}^{(k)}$ and condition (W4) we have

$$
\frac{1}{h^{2}} m_{0}^{(k)}(\mathrm{Id}+h G) \leq \frac{1}{h^{2} k^{2}} \int_{Z_{0}^{(k)}} W_{0}(\operatorname{Id}+h G) \mathrm{d} x=\frac{1}{2} Q_{0}(G)+o\left(h^{2}\right)
$$

and (7.20) follows by passing to the $\limsup _{h}$ on both sides.

Proof of (7.21) by contradiction: Suppose that there exist $k \in \mathbb{N}, G \in \mathcal{G}$ and a vanishing sequence of positive numbers $\left(h_{j}\right)_{j \in \mathbb{N}}$ such that

$$
\lim _{j \rightarrow \infty} \frac{1}{h_{j}^{2}} m_{0}^{(k)}\left(\operatorname{Id}+h_{j} G\right)=0 .
$$

By definition of $m_{0}^{(k)}$ there exists a sequence $\left(\varphi_{j}\right) \subset X_{0}^{(k)}$ such that

$$
\lim _{j \rightarrow \infty} \frac{1}{h_{j}^{2}} \int_{Z_{0}^{(k)}} W_{0}\left(\mathrm{Id}+h_{j}\left(G+\nabla \varphi_{j}\right)\right) \mathrm{d} y=0 .
$$

By appealing to the non-degeneracy condition (W3) and geometric rigidity (see Theorem 3.1) there exist rotations $R_{j}$ such that

$$
\lim _{j \rightarrow \infty} h_{j}^{-1}\left\|\operatorname{Id}+h_{j}\left(G+\nabla \varphi_{j}\right)-R_{j}\right\|_{L^{2}\left(Z_{0}^{(k)}\right)}=0 .
$$


Since $\varphi_{j}$ is periodic in its first and second variable, we get for $i=1,2$

$$
\begin{aligned}
h_{j}^{-1}\left|\left(\operatorname{Id}-R_{j}\right) e_{i}\right| & \leq h_{j}^{-1}\left|\left(\operatorname{Id}+h_{j} G-R_{j}\right) e_{i}\right|+\left|G e_{i}\right| \\
& =\left.h_{j}^{-1}|| Z_{0}^{(k)}\right|^{-1} \int_{Z_{0}^{(k)}}\left(\operatorname{Id}+h_{j}\left(G+\nabla \varphi_{j}\right)-R_{j}\right) e_{i} \mathrm{~d} x|+| G e_{i} \mid \\
& \leq h_{j}^{-1}||\left(\operatorname{Id}+h_{j}\left(G+\nabla \varphi_{j}\right)-R_{j}\right) e_{i} \|_{L^{2}\left(Z_{0}^{(k)}\right)}\left|Z_{0}^{(k)}\right|^{-1 / 2}+\left|G e_{i}\right|
\end{aligned}
$$

Hence, in virtue of (7.24), this implies $\left|\left(\operatorname{Id}-R_{j}\right) e_{i}\right| \leq c h_{j}$ for $i=1,2$ and a constant $c$ independent of $j$. By appealing to the property that $R_{j} \in S O(3)$, we find that $\left|\mathrm{Id}-R_{j}\right| \leq 2 c h_{j}$. Consequently, by (7.24) the sequence $\left(\nabla \varphi_{j}\right)$ is bounded in $L^{2}\left(Z_{0}^{(k)}\right)$. Now, we can proceed as in the proof of Theorem 1.1 (Step 2) and get

$$
\liminf _{j \rightarrow \infty} \frac{1}{h_{j}^{2}} m_{0}^{(k)}\left(\operatorname{Id}+h_{j} G\right) \geq \inf _{\varphi \in X_{0}^{(k)}} \frac{1}{k^{2}} \int_{Z_{0}^{(k)}} Q_{0}(\operatorname{sym}(G+\nabla \varphi)) \mathrm{d} y .
$$

By appealing to the periodicity properties of maps in $X_{0}^{(k)}$ and since $Q_{0}$ is positive definite on the subspace of symmetric matrices, we can easily derive a (non-optimal) lower bound; namely, the right hand side is bounded from below by

$$
c^{(k)}\left|G:\left(e_{2} \otimes e_{2}\right)\right|^{2} \quad \text { for some positive constant } c^{(k)}>0 .
$$

For $G \in \mathcal{G}$ this expression is strictly positive - in contradiction to (7.23); thus, (7.21) follows.

The argument for (7.22) is more subtle and relies on the observation that $I_{0}^{(k)}$ can be written as the elastic energy of a membrane occupying the slender domain

$$
\Omega^{(k)}:=\omega \times\left(0, \frac{1}{2 k}\right), \quad \omega:=(0,1)^{2} .
$$

To make this precise, define the functional

$$
J^{(k)}(u):=\frac{1}{\left|\Omega^{(k)}\right|} \int_{\Omega^{(k)}} W_{0}(\nabla u) \mathrm{d} z, \quad u \in W^{1,2}\left(\Omega^{(k)} ; \mathbb{R}^{3}\right),
$$

and notice that with the scaling $\hat{u}(x)=k u(x / k)$ we have

$$
J^{(k)}(u)=\frac{1}{2 k^{2}} \int_{Z_{0}^{(k)}} W_{0}(\nabla \hat{u}) \mathrm{d} x .
$$

As a consequence it follows that

$$
\begin{aligned}
& \frac{m_{0}^{(k)}(\operatorname{Id}+h G)}{2} \leq \inf \left\{J^{(k)}(u): u \in X_{\mathrm{Id}+h G}^{(k)}\right\}, \\
& \quad \text { where } X_{F}^{(k)}:=\left\{u \in W^{1,2}\left(\Omega^{(k)} ; \mathbb{R}^{3}\right), u(z)=F z \text { on } \partial \omega \times\left(0, \frac{1}{2 k}\right)\right\} .
\end{aligned}
$$

In 14] it is shown that $J^{(k)} \Gamma$-converges as $k \rightarrow \infty$ to a membrane energy which is zero for short maps, i.e. for deformations $u: \omega \rightarrow \mathbb{R}^{3}$ with $\nabla u^{\mathrm{T}} \nabla u \leq \mathrm{Id}$. Since $(\operatorname{Id}+h G)^{\mathrm{T}}(\operatorname{Id}+h G) \leq \operatorname{Id}$ for all $G \in \mathcal{G}$ and sufficiently small $h>0$, it is natural to prove (7.22) by appealing to a suitable recovery sequence. For our purpose it is not necessary to derive the precise form of the limiting functional. Indeed, the following is sufficient: For all $G \in \mathcal{G}$ and $0<h \ll 1$ there exists a sequence $\left(u^{(k)}\right)$, $u^{(k)} \in X_{\mathrm{Id}+h G}^{(k)}$ such that

$$
\limsup _{k \rightarrow \infty} J^{(k)}\left(u^{(k)}\right)=0
$$


In combination with (7.25) this clearly proves the assertion. The existence of such a sequence is shown for instance in [7, 14].

Step 4. (Conclusion). Argument for a). We only prove (W3'). Since $W_{0}$ is nonnegative and non-degenerate (see condition (W2)), we have for all $\psi \in W_{\text {per }}^{1,2}\left(Y ; \mathbb{R}^{3}\right.$ )

$$
\int_{Y} W(y, F+\nabla \psi) \mathrm{d} y \geq \int_{Y_{0}} W_{0}(F+\nabla \psi) \mathrm{d} y \geq c^{\prime} \int_{Y_{0}} \operatorname{dist}^{2}(F+\nabla \psi, S O(3)) \mathrm{d} y .
$$

Here and below, $c^{\prime}, c^{\prime \prime}, c^{\prime \prime \prime}$ are positive constants that only depend on $W_{0}$ and on the geometry of $Y_{0}$. By geometric rigidity (see Theorem 3.1) we get (for some $R \in S O(3))$

$\int_{Y} W(y, F+\nabla \psi) \mathrm{d} y \geq c^{\prime} \int_{Y_{0}}|F+\nabla \psi-R|^{2} \mathrm{~d} y \stackrel{(\star)}{\geq} c^{\prime \prime}|F-R|^{2} \geq c^{\prime \prime \prime} \operatorname{dist}^{2}(F, S O(3))$.

Inequality $(\star)$ is valid, since

$$
\inf _{G, \psi} \int_{Y_{0}}|G+\nabla \psi(y)|^{2} \mathrm{~d} y>0
$$

where the infimum is taken over all $G \in \mathbb{M}^{3}$ with $|G|=1$ and $\psi \in W_{\text {per }}^{1,2}\left(Y ; \mathbb{R}^{3}\right)$ satisfying $\int_{0}^{1} \int_{0}^{1} \partial_{3} \psi \mathrm{d} y_{2} \mathrm{~d} y_{1}=0$.

Proof of b) by contradiction. Suppose that there exists $F \in \mathbb{M}^{3}$ and $k \in \mathbb{N}$ such that $W_{\text {hom }}^{(k)}(F)=0$. Then (7.11) implies that $m_{0}^{(k)}(F)=m_{\rho}^{(k)}(F)=0$. As in the proof of (7.13), we find that $m_{0}^{(k)}(F)=0$ implies $\left|F e_{1}\right|=1$, while $m_{\rho}^{(k)}(F)=0$ implies $\left|F e_{1}\right|>1$. But this is a contradiction.

Statement c) is a direct consequence of (17.13), the splitting in Step 1 and the property that $W(y, \mathrm{Id})=W_{0}(\mathrm{Id})=0$ for $y \in Y_{0}$.

Statement d) follows from (7.13), the splitting in Step 1 and (7.20).

Proof of e). Let $G \in \mathcal{G}$, i.e. $G=-g\left(e_{2} \otimes e_{2}\right)$ where $g \in(0,1)$. Note that by Step 1 we have $W_{\text {hom }}^{(k)}(\mathrm{Id})=m_{0}^{(k)}(\mathrm{Id})+m_{\rho}^{(k)}(\mathrm{Id})=m_{\rho}^{(k)}(\mathrm{Id})$ and therefore

$$
W_{\text {hom }}^{(k)}(\operatorname{Id}+h G)-W_{\text {hom }}^{(k)}(\operatorname{Id})=m_{0}^{(k)}(\operatorname{Id}+h G) .
$$

With (7.20) and (7.21) we obtain

$$
0<\liminf _{h \downarrow 0} \frac{m_{0}^{(k)}(\operatorname{Id}+h G)}{h^{2}} \leq \limsup _{h \downarrow 0} \frac{m_{0}^{(k)}(\operatorname{Id}+h G)}{h^{2}} \leq \frac{1}{2} Q_{0}(G) .
$$

Hence, if $\sigma^{(k)} \in \mathbb{M}^{3}$ fulfills (7.8) it must satisfy $\left\langle\sigma^{(k)}, G\right\rangle=0$ and we get

$$
\liminf _{h \downarrow 0} \frac{1}{h^{2}}\left(W_{\text {hom }}^{(k)}(\operatorname{Id}+h G)-W_{\text {hom }}^{(k)}(\mathrm{Id})-h\left\langle\sigma^{(k)}, G\right\rangle\right)>0 .
$$

On the other side, the combination of (17.26) and (7.22) implies that

$$
\lim _{h \downarrow 0} \frac{1}{h^{2}} W_{\mathrm{hom}}^{(\mathrm{mc})}(\mathrm{Id}+h G)=0,
$$

which completes the proof.

\section{ACKNOWLEDGEMENTS}

We thank S. Conti for very inspiring discussions. The second author gratefully acknowledges the hospitality of the Hausdorff Center for Mathematics in Bonn, as part of this work was accomplished there. 


\section{REFERENCES}

[1] Abeyaratne, R., Triantafyllidis, N.: An investigation of localization in a porous elastic material usign homogenization theory. J. Appl. Mech. 51, 481-486 (1984)

[2] Allaire, G.: Homogenization and two-scale convergence. SIAM J. Math. Anal. 23(6), 1482$1518(1992)$

[3] Baía, M., Fonseca, I.: The limit behavior of a family of variational multiscale problems. Indiana Univ. Math. J. 56(1), 1-50 (2007)

[4] Braides, A.: Homogenization of some almost periodic coercive functional. Rend. Accad. Naz. Sci. Detta XL, V. Ser., Mem. Mat. 9, 313-322 (1985)

[5] Braides, A., Defranceschi, A.: Homogenization of multiple integrals. Oxford Lecture Series in Mathematics and its Applications. 12. Oxford: Clarendon Press. xiv (1998)

[6] Braides, A., Solci, M., Vitali, E.: A derivation of linear elastic energies from pair-interaction atomistic systems. Netw. Heterog. Media 2(3), 551-567 (2007)

[7] Conti, S.: Low-energy deformations of thin elastic sheets: isometric embeddings and branching patterns. Habilitation thesis, Universität Leipzig (2003)

[8] Dal Maso, G.: An introduction to $\Gamma$-convergence. Progress in Nonlinear Differential Equations and their Applications. 8. Basel: Birkhäuser. xiv (1993)

[9] Dal Maso, G., Negri, M., Percivale, D.: Linearized elasticity as $\Gamma$-limit of finite elasticity. Set-Valued Analysis 10, 165-183 (2002)

[10] Francfort, G., Murat, F.: Homogenization and optimal bounds in linear elasticity. Arch. Ration. Mech. Anal. 94, 307-334 (1986)

[11] Francfort, G., Murat, F., Tartar, L.: Homogenization of monotone operators in divergence form with $x$-dependent multivalued graphs. Ann. Mat. Pura Appl. 4, 631-652 (2009)

[12] Friesecke, G., James, R.D., Müller, S.: A theorem on geometric rigidity and the derivation of nonlinear plate theory from three-dimensional elasticity. Commun. Pure Appl. Math. 55(11), 1461-1506 (2002)

[13] Geymonat, G., Müller, S., Triantafyllidis, N.: Homogenization of nonlinearly elastic materials, microscopic bifurcation and macroscopic loss of rank-one convexity. Arch. Ration. Mech. Anal. 122(3), 231-290 (1993)

[14] LeDret, H., Raoult, A.: The nonlinear membrane model as a variational limit of nonlinear three-dimensional elasticity. J. Math. Pures Appl. 73, 549-578 (1995)

[15] Marcellini, P.: Periodic solutions and homogenization of non linear variational problems. Ann. Mat. Pura Appl., IV. Ser. 117, 139-152 (1978)

[16] Müller, S.: Homogenization of nonconvex integral functionals and cellular elastic materials. Arch. Ration. Mech. Anal. 99, 189-212 (1987)

[17] Neukamm, S.: Homogenization, linearization and dimension reduction in elasticity with variational methods. Ph.D. thesis, Technische Universität München (2010)

[18] Olejnik, O., Shamaev, A., Yosifyan, G.: Problèmes d'homogénéisation pour le système de l'élasticité linéaire à coefficients oscillant non-uniformément. (Problems of homogenization for linear elasticity system with non-uniformly oscillating coefficients). C. R. Acad. Sci., Paris, Sér. I. 298, 273-276 (1984)

[19] Schmidt, B.: On the derivation of linear elasticity from atomistic models. Netw. Heterog. Media 4(4), 789-812 (2009). DOI 10.3934/nhm.2009.4.789

[20] Tartar, L.: Cours peccot au collège de france. Paris, 1977

[21] Tartar, L.: The general theory of homogenization. A personalized introduction. Lecture Notes of the Unione Matematica Italiana 7. Berlin: Springer. xvii (2009)

[22] Triantafyllidis, N., Maker, B.: On the comparison between microscopic and macroscopic instability mechanisms in a class of fiber-reinforced composites. J. Appl. Mech. 52, 794-800 (1985)

[23] Zhang, K.: Quasiconvex functions, $S O(n)$ and two elastic wells. Ann. Inst. Henri Poincaré, Anal. Non Linéaire 14 6, 759-785 (1997)

(Stefan Müller) Hausdorff Center for Mathematics, Institute for Applied Mathematics, Universität Bonn, Endenicher Allee 60, 53115 Bonn, Germay

E-mail address: stefan.mueller@hcm.uni-bonn.de 
(Stefan Neukamm) Zentrum Mathematik / M6, Technische Universität München, Boltzmannstrasse 3,85748 Garching bei München, Germany

Current address: Max Planck Institute for Mathematics in the Sciences, Inselstraße 22, 04103 Leipzig, Germany

E-mail address: neukamm@mis.mpg.de 